\title{
Microscale modeling of particle impact on TPS materials during high-speed entry
}

\author{
Savio J. Poovathingal* and Hailong Chen ${ }^{\dagger}$ \\ Department of Mechanical Engineering, University of Kentucky, Lexington, KY 40506.
}

\begin{abstract}
Micron-sized particles suspending in planetary atmospheres can damage thermal protection systems (TPS) during entry of space capsules into planetary bodies. TPS materials are complex heterogeneous carbon composites, where the microstructure of the composite can play a pivotal role in the propagation of the damage caused by the impact. Here, we present an application of a novel computational technique called the lattice particle method to understand the initiation and growth of craters formed on TPS materials upon impact by particles. The simulations are initially compared against experiments that used borosilicate as the impacting particle and fused silica as the target surface. The simulations reproduce the damage profiles, diameters, and depths of the craters being formed on the target silica surface. A parametric study is then performed by varying the fracture strength of the target surface and the impacting particle. It is found that the profiles of the damaged region on the silica surface primarily depends on the fracture strength of the silica surface, and not the impacting particle. The simulations are extended to model the damage of porous carbon composites that are used as TPS materials. Microstructures of carbon composites are generated using an in-house code that has been shown to reproduce features of the real material in past studies. While the crater depth on the fused silica surface was within $38 \%$ irrespective of the fracture strength of the particle, the damaged depth changes by at least an order of magnitude when a carbon composite surface is used and the fracture strength of the impacting particle is varied. Finally, the influence of damage on the effective permeability is computed using the direct simulation Monte Carlo technique. The maximum increase in the permeability force for the damaged microstructures is found to be $20 \%$, which suggests that the crater created in the damaged microstructure does not significantly influence the path traversed by gases percolating through the porous TPS material.
\end{abstract}

\footnotetext{
*Assistant Professor, saviopoovathingal@uky.edu, AIAA Member.

†Assistant Professor, hailong.chen@uky.edu, AIAA Member.
} 


\section{Introduction}

Atmospheric and surface events cause small dust particles to suspend in the atmosphere of planetary systems. For example, irregular dust storms are a common occurrence on Mars that leave solid particles in suspension in the atmosphere for a long time [1-3]. Suspended aerosol particles are observed in the upper atmospheres of Earth [4], and ice particles are observed in the atmosphere of Uranus and Neptune (ice giants) [5]. The entry/re-entry of space capsules are characterized by speeds exceeding Mach 20. During the high-speed descent, particles in the atmosphere are transported at high velocities crashing into the space capsule. During a typical entry trajectory into the Mars atmosphere, particles ranging from 1-10 $\mu \mathrm{m}$ are encountered by the capsule with the particles striking the capsule at velocities ranging from 1-4 km/s [6]. Although reusable, ceramic-based, thermal protection system (TPS) materials (TPS is also referred to as a heat shield material in this article) can be used for lifting trajectories (e.g. the space shuttle), higher entry speeds encountered during Mars entry, or Earth re-entry returning from the Moon or Mars require ablative TPS [7]. Ablative TPS are typically porous carbon composites containing carbon fibers or weaves into which phenolic resin is infused. These materials relieve heat load through a number of processes, including absorption processes such as thermochemical decomposition of the filler resin and sublimation, as well as heat rejection processes through modification of the high-temperature boundary layer through transpiration of ablation products, and systematic sacrificial removal of hot material through ablation [8]. The high kinetic energy attained by the particles can damage the TPS material during impact, potentially introducing new failure modes through a change in the effective material properties or through enhancement of thermochemical gas-surface processes. Additionally, the impact creates craters on the surface that increases the recession of the TPS material [6, 9].

The erosion of TPS materials by particle impacts can be investigated through a combination of computational fluid dynamics (CFD) techniques, and the recession modeled through material response codes. Ref. [6] provides a review of the current state-of-the-art approaches available within the community to investigate the impact of particles on entry vehicles. A brief summary is provided here. The transport of dust particles to the surface is modeled using either a one-way or two-way coupled particle-fluid simulations. In one-way coupled simulations, the flowfield is computed first, and the particle trajectories integrated using the underlying flowfield and a drag model. It is assumed that the particles are small and disperse enough such that the particles do not influence the flow around the capsule. This approach was taken by Papadopoulos et al. [9] and Palmer et al. [10] by using a thin-layer, Navier-Stokes code to obtain the flowfield. In two-way coupled simulations, the fluid field and the particle field are modeled simultaneously, and the particle and the flowfield influence each other. Elangovan and Cao [11] and Saito et al. [12] have performed two-dimensional CFD analysis using a pure Eulerian approach for both the particles and the flowfield where the particle trajectory and fluid dynamic equations were solved together using the same grid. Recently, a Lagrangian particle tracking methodology using a high-order discontinuous Galerkin scheme by Palmer et al. [10] and others [13, 14] was used to compute coupled particle-fluid simulations of high-speed dusty flows over blunt bodies. Care must be taken when using a full Eulerian 
approach and in the choice of the drag and heat transfer model because the dust particles are small enough such that the flow around the particle is usually in the non-continuum regime [9].

Erosion of the char layer is the most evident effect of dust clouds encounter. The erosion of the material can be modeled by material response codes like Charring Materials Thermal Response and Ablation (CMA) [15, 16], Fully Implicit Ablation and Thermal (FIAT) [17], 3dFIAT [18, 19], Two-dimensional Implicit Thermal response And ablatioN (TITAN) [20], CHarring Ablator Response (CHAR) [21-23], Modeling of Pyrolysis and Ablation Response (MOPAR) [24-26], Kentucky Aerothermodynamics and Ablation Response System - Material Response (KATS-MR) [27--29], Porous-material Analysis Toolbox based on OpenFOAM (PATO) [30], and Icarus [31]. To account for erosion by impact of particles, the damaged region is captured in material response codes by creating holes of the appropriate diameter and depth in the material response mesh. Therefore, material response codes require a closure model that provides this information. This model would provide information about the damaged region (example crater diameter and penetration depth) based on various particle parameters such as diameter, impacting velocity, and density.

Papadopoulos et al. [9] developed a model that focused on Martian dust storms and the model is based on experimental data that used silica as the target surface [32]. Since the Martian dust particle composition is largely silicate-based, the pyrex projectile data from Ref. [32] was used to develop the Martian damage model. Spherical projectiles were used and based on this data, the correlations shown in Eqns. 1 and 2 were developed for crater diameter $\left(\mathrm{D}_{c}\right)$ and penetration depth $(p)$.

$$
\begin{aligned}
\frac{D_{c}}{d_{p}} & =0.113 v_{p}^{0.667} \\
\frac{p}{d_{p}} & =0.3016 d_{p}^{0.4} v_{p}^{0.667}
\end{aligned}
$$

where, $v_{p}$ and $d_{p}$ are velocity and diameter of the dust particle, respectively. In this model, it is assumed that the damage region is cylindrical with a diameter of $\mathrm{D}_{c}$ and length $p$. The experiments on the fused silica surface were primarily aimed at understanding the potential failure of windows on the Space Shuttle [32]. Recently, Palmer et al. developed new correlations for Norcoat Liége, a carbon-based ablative TPS [6]. Norcoat Liége is comprised of cork into which phenolic resin is infused. The empirical correlations for Norcoat Liége is shown in Eqns. 3 and 4 The particles used in the experiments for Norcoat Liége were boron and alumina, which has properties that are likely different from a Martian dust particle. 


$$
\begin{aligned}
D_{c} & =0.00056 \rho_{p}^{0.62} d_{p}^{1.04867} v_{p}^{0.667} \\
p & =0.00028 \rho_{p}^{0.62} d_{p}^{1.04867} v_{p}^{0.667}
\end{aligned}
$$

where, $v_{p}, d_{p}$, and $\rho_{p}$ are velocity, diameter, and density of the particle, respectively. There is no experimental data available for the impact of a Martian-like dust particle striking a highly porous anisotropic composite such as phenolic impregnated carbon ablator (PICA). Since dust-impact erosion models for a PICA-like composite is not available, the fused silica or Norcoat Liége data (Eqns. 1 4 , is is currently used to model the erosion process [6]. Further, these erosion models do not capture the influence of damage on effective material properties such as thermal conductivity, material permeability, coefficient of thermal expansion, and radiative coefficients. Finally, an analysis on the effect of damage on thermochemical processes such as in-depth diffusion of gases into the material, blowing of pyrolysis gases, and the thermostructural response of the material is not available.

In this work, we aim to build a fundamental understanding of the damage occurring on a TPS material when a high-speed particle strikes it. We simulate the impact process at the microscale (also referred to as mesoscale), $O\left(10^{3}-10^{4} \mu \mathrm{m}\right)$, using a novel technique called the lattice particle method (LPM). LPM simulates the impact of a particle on the microstructure of a material capturing the damage profiles formed on the surface. The damaged microstructure can then be used to explore the effects of damage on other thermochemical properties and processes. This article primarily focuses on validation and identifying key features that need to be captured during the damage process. In order to explore an aspect of the change in material properties, effective permeability is computed for the damaged microstructure and compared against the values for the virgin microstructure. This property is chosen because microscale flow computations of effective permeability has been validated against experimental data [33, 34].

\section{Computational Methods}

There are three computational techniques used in this work. First, the microstructures of carbon composites are generated using a Fibergen code; second, the damage caused by an impacting dust particle is simulated using a lattice particle method (LPM); and third, a flow solver is used to compute effective material permeability for the damaged microstructure. All three methods are discussed below.

\section{A. Generation of Microstructures}

Fibergen generates cylindrical fibers that reproduces the microstructure of FiberForm, which is the base carbon material that forms PICA. In the Fibergen code, the user can control the distribution of three-dimensional orientations, fiber diameters, bulk porosity, fiber density, and material density. Resin is infused into the sample through a stochastic 
approach [34]. In the manufacturing of the composite, the resin is infused into the microstructure of FiberForm which bonds to the carbon fibers in a random manner. Since the resin randomly infuses into the composite, a similar approach is taken. To incorporate the resin, the fibers are initially generated. The box containing the fibers is discretized to a desired resolution and the volume occupied by the fibers in each cell is computed using the cut-cell technique [35]. A discretized cell within the three-dimensional box is chosen randomly. Cells that are completely inside the fiber or that do not contain any fiber (no cut-volume) are ignored and a new cell is chosen until a cell with partially occupied fiber is selected. The volume occupied by the fiber inside the cell is known through the cut-cell technique [35] and the resin is added to the remaining volume. Currently, it is assumed that the entire remaining volume of the cell is occupied by the resin. Resin is added until the density or porosity of the entire composite reaches the target value. The microstructures can be obtained in both stl and voxel format. In this article, the microstructures of the full composite is exported in the voxel format. The voxel format identifies the three phases, namely fiber, resin, and voids using unique integer values. A sample microstructure generated in stl and voxel format is shown in Fig. 1. In Fig. [1b, red pixels represent the resin that is bonded to the underlying base structure of fibers, represented in black. The ability of Fibergen to reproduce material features and effective material properties (permeability and radiative coefficients) has been previously validated [7, 34, 36]. Additional details of the Fibergen code can be found in Refs. [33, 34].

A representative elementary volume (REV) of $200^{3} \mu m\left(0.2^{3} \mathrm{~mm}\right.$ ) microstructural sample is generated with a voxel resolution of $1.25 \mu \mathrm{m}$ resulting in a total of 5,994,531 voxels (179 in x, 183 in $\mathrm{y}$ and $\mathrm{z}$ ). The FiberForm density and the full composite density (FiberForm + resin) is set to 180 and $210 \mathrm{~kg} / \mathrm{m}^{3}$, respectively, which results in a net porosity of $\sim 0.8$. These values provide the best agreement with permeability measurements based on a previous study [34].

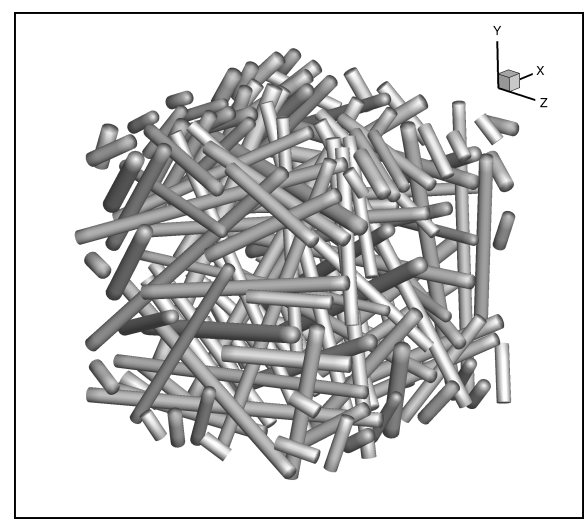

(a) Surface mesh in STL format.

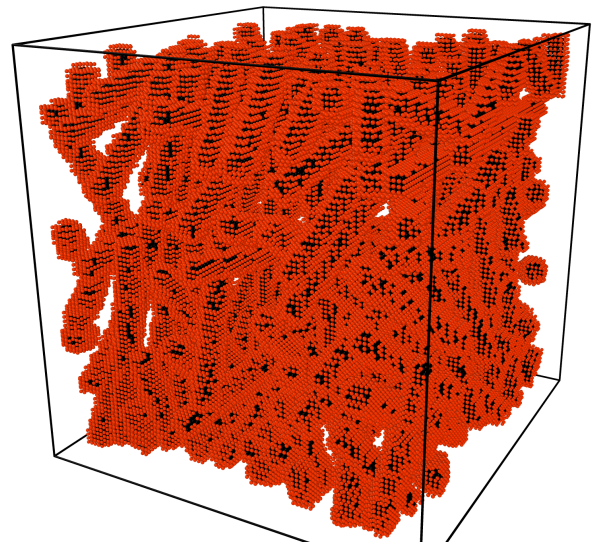

(b) Surface mesh in voxel format at a resolution of $2.5 \mu \mathrm{m}$.

Fig. 1 Microstructures generated using the Fibergen code. The figure on the left contains only fibers, while the figure on the right is comprised of both both fibers and resin. In Fig. 1b, red and black represent the resin and fiber, respectively. 


\section{B. Lattice Particle Method}

Lattice particle method (LPM) is a recently developed non-local mesh-free method for modeling the failure of both composite and polycrystalline materials at the mesoscale [37-40]. For modeling composite materials, LPM discretizes the material microstructure into voxels. A voxel interacts with neighboring voxels up to the second nearest neighbors via a bond-like force (see Fig. 2). As a result, LPM does not require mesh generation that is usually considered the most difficult part in mesh-based approaches for complex microstructures.

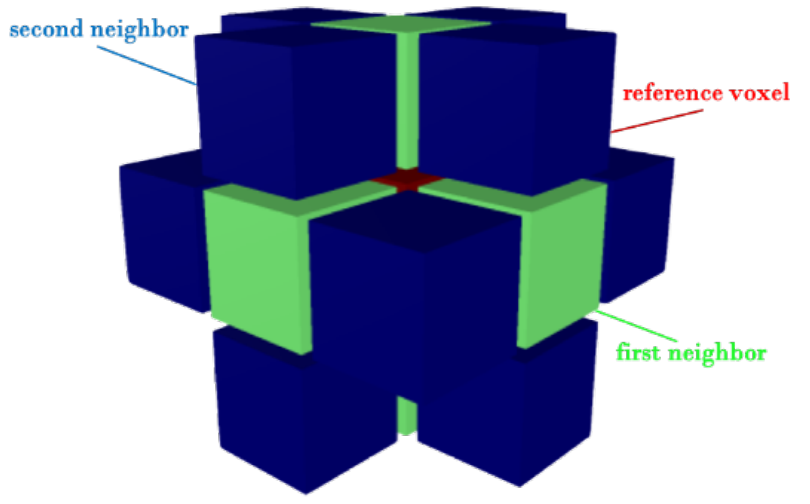

Fig. 2 Non-local interaction between voxels in the LPM method.

In LPM [39], the potential energy of a voxel $I$ for isotropic materials has the following form

$$
U^{I}=\frac{1}{2} k_{l} \sum_{J=1}^{N^{I}}\left(d l^{I J}\right)^{2}+\frac{1}{2} k_{n}\left(\sum_{J=1}^{N^{I}} d l^{I J}\right)^{2}
$$

where $d l$ is bond elongation, $N$ is the number of interacting neighboring voxels, $k_{l}$ and $k_{n}$ are the model local and nonlocal parameters that is determined using the energy equivalency between LPM and its continuum counterpart. In terms of material constants, the model parameters $k_{l}$ and $k_{n}$ can be found as follows:

$$
k_{l}=\frac{a E}{1+v}
$$

$$
k_{n}=\frac{a E(4 v-1)}{18(1+v)(1-2 v)},
$$

with $E$ being the Young's modulus, $v$ is the Poisson's ratio, and $a$ is the voxel size [39].

Finally, the force between two interacting voxels $I$ and $J$ can be determined by differentiating the potential energy 
with respect to the bond elongation as follows:

$$
\mathbf{f}^{I J}=\frac{\partial\left(U^{I}+U^{J}\right)}{\partial d l^{I J}} \mathbf{n}^{I J}=\left(k_{l} d l^{I J}+\frac{1}{2} k_{n}\left(\sum_{M=1}^{N^{I}} d l^{I M}+\sum_{M=1}^{N^{J}} d l^{J M}\right)\right) \mathbf{n}^{I J}
$$

with $\mathbf{n}^{I J}$ being the unit normal vector pointing from voxel $I$ to its neighbor $J[39]$.

In LPM, crack nucleation and growth are modeled using bond breakage between otherwise interacting voxels. A bond is considered as broken and cannot sustain further tensile force when a bond-based quantity such as elongation exceeds its critical value [37]. Continuum-like measures, such as deformation gradient, strain and stress tensors can be constructed in LPM [41]. These constructed continuum-like measures can be used to incorporate continuum-mechanics-based failure models, such as fracture and fatigue damage theories to develop bond-based failure criterion in LPM. A typical critical elongation based failure criterion can be written as

$$
d l_{i}^{c}=\alpha_{i} l_{i}^{0}
$$

where $i=1,2$ for bonds connecting with the first and the second nearest neighbors, $l_{i}^{0}$ is the original length of a bond, and $\alpha_{i}$ is the critical elongation coefficient that can be related to material fracture strength via calibration with experimental data [42]. For isotropic solids, the following relationship exists between the two critical elongation coefficients (for the first and the second nearest neighbors) [42] as

$$
\alpha_{1}=\sqrt{2} \alpha_{2}
$$

For brittle materials, the critical elongation coefficient for the first nearest neighbors $\alpha_{1}$ was calibrated based on the material fracture strength, voxel size and elastic constants [42], which can be written as

$$
\alpha_{1}=0.625 \frac{a \sigma_{f}}{k_{l}}
$$

where $\sigma_{f}$ is the material fracture strength.

To visualize the LPM simulation results, a damage parameter is defined for each voxel based on the status of all bonds associated with it as 


$$
D=\frac{N_{b b}}{N_{t b}}
$$

where $N_{b b}$ is the number of broken bonds initially connected with the voxel and $N_{t b}$ is the number of current total bonds connected with the voxel.

A prototypical image to model the damage process in the simulation is shown in Fig 3 The figures represent voxels in the domain. The blue voxels represent the target structure, while the black voxels represent the impacting particle. The voxels are colored by the damage values (Eqn. 12). Initially, there is no damage and all voxels have equal number of connected bonds (Fig. 3a). As the particle strikes the target, forces on the voxels from the new interactions change the bond stretch resulting in the initiation of damage. This is seen in Fig. 3b, where the voxels on the surface are beginning to break away from the underlying material as the bonds are cleaved. As the simulation proceeds, more bond stretches reach the critical value resulting in bond breakage and eventually the voxel is completely disconnected from the surface. In addition, the particle itself can be damaged in a similar manner if the voxels of the particle move away from each other exceeding the critical threshold. This process is explored in Sec. III.A.

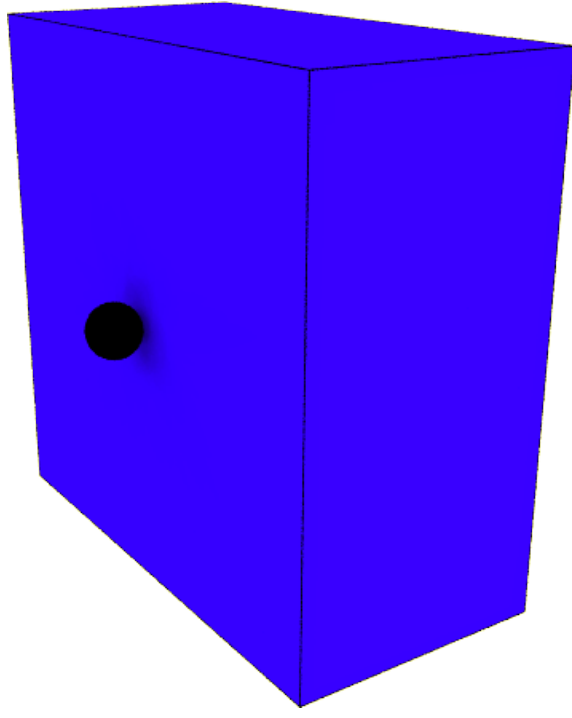

(a) Initialization of LPM simulations.

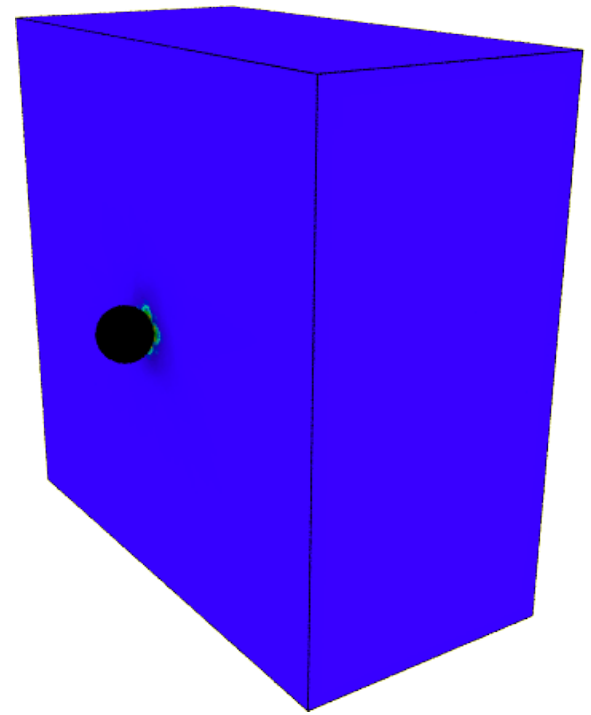

(b) Snapshot at the initiation of damage.

Fig. 3 Schematic of particle impact simulations using the LPM method where the voxels are colored by the damage factor.

\section{Fundamental mechanical properties of each phase}

LPM requires intrinsic mechanical properties of each phase within the material to determine the local $\left(k_{l}\right)$ and non-local parameters $\left(k_{n}\right)$ before it can be applied to model material failure. The required mechanical properties include 
the mass density, Young's modulus, Poisson ratio, and the fracture strength. Fused silica and carbon composites are used as target surfaces and projectiles (particles) comprised of Pyrex is used in this study. Therefore, the mechanical properties of fused silica is needed for the validation study (see Sec. [III.A), and the mechanical properties of the carbon fiber and the phenolic resin are needed for simulations of the carbon composite. The mechanical properties of Pyrex is needed to model the particle. The mechanical properties are shown in Table 1. The properties have been compiled from various sources. A handbook with properties for fused silica and Pyrex is not available since it varies significantly based on the manufacturer. Therefore, for both Pyrex and fused silica, we rely on manufacturer data sheets to obtain representative values. The sources for the properties of fused silica and Pyrex are given in the Appendix (Sec. V). The mass density, Young's modulus, and Poisson ratio appear to be consistent among all manufacturers for both fused silica and Pyrex. However, the fracture (tensile) strength of fused silica varies from 50 - $500 \mathrm{MPa}$, and that of the Pyrex is reported as either 80 or $280 \mathrm{MPa}$. In this work, the fracture strength of Pyrex is taken as $280 \mathrm{MPa}$, while simulations of fused silica with both 50 and $500 \mathrm{MPa}$ are performed (see Sec. IIII.A). The properties of carbon fiber could also vary through manufacturing processes. High-strength carbon fibers have fracture strength in the range of $690-1030 \mathrm{MPa}$ [43], while rayon-based fibers have a fracture strength of 330 - $900 \mathrm{MPa}$ [44, 45]. The properties of phenolic resin appear to be well-defined with lower variability compared to the fiber properties [46, 47].

Table 1 Fundamental mechanical properties of the various phases. See Sec. $V$ for sources used for compiling the data of fused silica and Pyrex.

\begin{tabular}{|c|c|c|c|c|}
\hline & $\begin{array}{c}\text { Fused silica } \\
\text { (quartz) }\end{array}$ & $\begin{array}{c}\text { Carbon } \\
\text { fiber }\end{array}$ & $\begin{array}{c}\text { Phenolic } \\
\text { resin }\end{array}$ & $\begin{array}{c}\text { Pyrex } \\
\text { (Borosilicate glass) } \\
\text { Particle }\end{array}$ \\
\hline Density $\left(\mathrm{kg} / \mathrm{m}^{3}\right)$ & 2203 & 1400 & 1200 & 2230 \\
\hline Young's modulus $(\mathrm{GPa})$ & 72 & 80 & 4.43 & 67 \\
\hline Poisson ratio & 0.17 & 0.27 & 0.26 & 0.2 \\
\hline Fracture strength (MPa) & $50-500$ & $330-1030$ & 41.17 & $80 / 280$ \\
\hline
\end{tabular}

\section{Flow Computations}

The flow computations are performed using the the open-source DSMC solver, Stochastic Parallel Rarefied-gas Time-accurate Analyzer (Sparta) [48-50] with in-house enhancements [34]. Sparta uses a multi-level Cartesian mesh to track and collide particles. Surfaces can be identified in two ways. A set of triangles representing the surfaces is directly read into Sparta (referred to as explicit mode in Sparta) or a set of voxels are converted to a closed surface with triangles using the marching cube algorithm (referred to as implicit mode in Sparta) [48, 51]. The triangles are sorted (for marching cube, sorting is not required) and the flow volume in each cell is computed using the cut-cell technique. In this work, surfaces are imported as voxels. The standard ray-tracing approach is used to move the simulation particles.

Sparta is used to compare effective permeability of virgin and damaged microstructures. To compute effective 
permeability, the microstructure is incorporated into the center of the simulated domain and symmetric boundary conditions are applied in the two spanwise directions ( $\mathrm{y}$ and $\mathrm{z}$ ). The incorporation of the microstructure into the flow domain is shown in Fig. 4. Subsonic boundary conditions are applied along the flow direction. In the upstream boundary, pressure $\left(\mathrm{P}_{i n}\right)$ and temperature $\left(\mathrm{T}_{i n}\right)$ are specified. The inflow velocity $(\mathrm{u})$ is obtained using a simple zeroth order extrapolation, i.e. $\mathrm{u}=\mathrm{u}_{j}$, where $j$ refers to the value at the first interior cell. In the downstream boundary, the method of characteristics of Nance et al. [52] and Fang and Liou [53] is used. The open-source version of Sparta contains only the option of obtaining instantaneous quantities from the interior of the flow, while the in-house version uses the sub-relaxation technique. The DSMC method is able to simulate all relevant physics including convection, multi-component diffusion, gas-phase and gas-surface chemistry, and is accurate for flow conditions ranging from continuum to free-molecular using the same set of collision model parameters [7, 33, 34, 54]. With the decoupling of the surface and flow mesh, arbitrarily complex geometries can be imported into the Sparta solver. Therefore, it is an ideal technique to obtain effective material permeability of complex porous materials. More details can be found in Refs. [7, 33, 34].

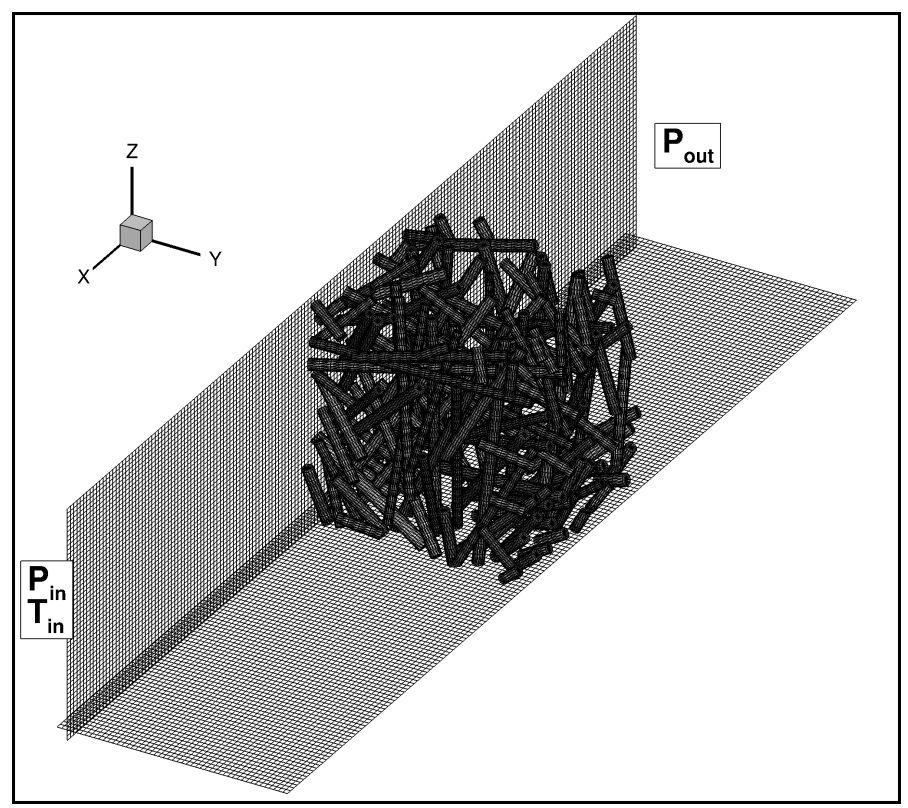

Fig. 4 Incorporation of the microstructure into the flow domain to compute effective material permeability.

The simulations are run until steady state and a permeability force $(\mathrm{F})$ is computed using Eqn. 13.

$$
F \equiv \frac{\mu \dot{m} T R L}{A M \Delta P}
$$

In Eqn. 13 . F is the permeability force, $\mu$ is the viscosity, $\dot{m}$ is the mass flow rate, $\mathrm{T}$ is the temperature, $\mathrm{R}$ is the universal gas constant, $\mathrm{L}$ is the length of the sample, $\mathrm{A}$ is the area, $\mathrm{M}$ is the molecular mass, and $\Delta P$ is the final pressure difference 
across the sample. A power law for viscosity is obtained using the variable hard sphere (VHS) model. Air is used as the gas mixture for the permeability computations. The reference diameter $\left(\mathrm{d}_{\text {ref }}\right)$ and VHS exponent $(\omega)$ for oxygen and nitrogen are as follows; $\mathrm{O}_{2}: d_{\text {ref }}=3.96 \times 10^{-10}, \omega=0.77$, and $\mathrm{N}_{2}: d_{\text {ref }}=4.17 \times 10^{-10}, \omega=0.74$.

\section{Results}

\section{A. Comparison of LPM Simulations with Experimental Data}

We begin with comparison of the damaged surface generated using the LPM method with past experiments. Specifically, we compare with experiments performed in 1970 for the space shuttle program by Flaherty [32]. In these experiments, projectiles were shot using a magnum-gun and velocity of the projectile measured through interrupting light beams in the path. A special technique was developed to estimate the internal damage caused by the impact. The fused silica target was sectioned near the crater, and the resulting edge was viewed under a microscope with bottom lighting. A cross-sectional view of the pulverized zone of the crater and a shadowgraph of the internal cracks and fissures were obtained through this approach. Various sections were observed by focusing at different planes through the crater and the fissures appeared black or various shades of gray at the boundary interface. Through this approach, Flaherty [32] quantified various damage regions on fused silica. The erosion model of Papadopoulos et al. [9] (Eqns. 1] and 2) was developed from this data.

In addition to comparing with the experiments of Flaherty, the influence of mechanical properties (see Sec. II.B.1) is assessed. Specifically, we compare the damage patterns for different combinations of the fracture strength of the fused silica target and the borosilicate particle. A fracture strength of 50 and $500 \mathrm{MPa}$ is used for fused silica. The fracture strength of the borosilicate particle is set to either $280 \mathrm{MPa}$ or a very high value, which is captured by not allowing the particle to fracture. The diameter and velocity of the particle is kept constant at $303 \mathrm{~m} / \mathrm{s}$ and $1 \mathrm{~mm}$, respectively. The top and cross-sectional view of these four combinations are shown in Figs. 5 and 6 The individual cases are referred by acronyms based on the simulation parameters. For example, the first case (Fig. 5a simulates a particle with a velocity of $303 \mathrm{~m} / \mathrm{s}$ (v303), fracture strength of $500 \mathrm{MPa}$ is used for fused silica (f500), and the fracture strength of the borosilicate particle is much higher than the target surface. Therefore, the particle is not damaged upon impact (nd). Therefore, it is referred to as case v303,f500,nd.

The top view of the fused silica surface for case v303,f500,nd is shown in Fig. 5a. The red color indicates voxels that have completely broken away from the base material leaving behind a crater on the surface. A diamond structure is formed on the surface under these conditions with the damaged region extending beyond the crater. The fracture appears to proceed along the two axes in the perpendicular direction. When the fracture strength of the target surface is lowered from $500 \mathrm{MPa}$ to $50 \mathrm{MPa}$, a petaloid structure is obtained with a much larger damaged region. This can be seen in Fig. $5 \mathrm{~b}$, which shows the top view of the surface with the same condition but with a modified fracture strength of 50 
$\mathrm{MPa}$ for the target silica surface. The green color indicates voxels that are significantly damaged but are still attached to the surface. Therefore, for case v303,f50,nd, the damaged region extends well beyond the crater (red voxels) that is formed on the surface. Although the crater formed in these two cases are not cylindrical, we define a crater diameter with the following definitions. For the simulation with a surface fracture strength of $500 \mathrm{MPa}$, we define a diameter that encompasses the red voxels depicted with a black circle in Fig. 5a Based on this definition, it is estimated that the crater diameter for the case with $500 \mathrm{MPa}$ is $1.94 \mathrm{~mm}$. For the case of $50 \mathrm{MPa}$, we define two circles to represent the inner (solid black lines) and outer crater regions (dashed black lines) as shown in Fig. $5 \mathrm{~b}$. The inner and outer diameters of the crater with a surface fracture strength of $50 \mathrm{MPa}$ is estimated to be 2.6 and $3.76 \mathrm{~mm}$, respectively. Flaherty estimates the damaged diameter for a particle striking at $303 \mathrm{~m} / \mathrm{s}$ to be $2.3 \mathrm{~mm}$.

Next, LPM simulations are performed for the same two cases but the particle fracture strength is set to $280 \mathrm{MPa}$ allowing the particle to be damaged upon reaching the failure criteria (referred with acronym d). The top view of the damaged surface with a surface fracture strength of $500 \mathrm{MPa}$ is shown in Fig. 5c, which shows a markedly different damaged profile. This difference in shape occurs because voxel chunks from the particle can now fly into neighboring voxels on the surface damaging them and creating additional failure. The damaged area is now circular with a diameter of $2.6 \mathrm{~mm}$, which is approximately $30 \%$ larger than the baseline case where the particle was not allowed to fracture (see Fig.5a). Although the damaged diameter for case v303,f500,d is larger, we note that this difference is fairly small considering that the damaged regions for case v303,f500,nd (Fig. 5a is not circular, and a rough diameter is estimated based on the red voxels. To assess this aspect further, simulations are performed where the surface fracture strength is set to $50 \mathrm{MPa}$ and the particle fracture strength is set to $280 \mathrm{MPa}$ (Fig. 5d). The comparison is similar to the case with a surface fracture strength of $500 \mathrm{MPa}$. The damage profile is markedly different with larger number of voxels removed, but the extent and diameter of the damaged region is similar to the case when the particle is not allowed to fracture.

Slices of the cross-sectional view of the damage with a fracture strength $500 \mathrm{MPa}$ and $50 \mathrm{MPa}$ without particle fracture and with particle fracture is shown in Figs. 6a, 6b, 6c, and 6d, respectively. The crater depth and diameters indicate that the fracture strength of the particle does not play a significant role in the damage of a solid silica surface. Additionally, in contrast to the experiments, the crater depth predicted by the LPM simulations are much smaller than the diameter of the crater when the fracture strength of the target surface is $500 \mathrm{MPa}$. A fissure depth can be estimated by defining the depth as the extent of red colored voxels inside the target. The estimated crater depths for a surface fracture strength of $50 \mathrm{MPa}$ are $2.62 \mathrm{~mm}$ (with particle fracture) and $3.06 \mathrm{~mm}$ (without particle fracture), respectively, while Flaherty estimates a crater depth of $2.7 \mathrm{~mm}$ [32]. Based on visual comparison (see Fig. 2 in Ref. [32]) and estimated crater depths of the cross-sectional slices from the LPM simulations, the fracture profiles obtained using a fracture strength of $50 \mathrm{MPa}$ for the target surface closely resembles the cross-sectional image and crater diameters and depths obtained in the experiments .

Given that it is unclear how the crater diameter and depth is defined by Flaherty [32], the intrinsic mechanical 
properties of the material used by Flaherty [32] is unknown, rough diameters and depths are estimated through voxel coloration (only red voxels are counted for crater depth and diameter) in LPM simulations, this level of agreement between the LPM simulations with a surface fracture strength of $50 \mathrm{MPa}$ and the experiments indicate that the LPM simulations are accurately capturing the damage process occurring on a solid target upon impact by a particle. Fine-grain quantitative distinctions based on experimental images from 50 years ago is avoided and only a qualitative picture is inferred in the comparisons here. Through this analysis, we primarily infer that the LPM simulations does predict damage in the same ballpark and that the damage patterns significantly depend on the fracture strength of the material and the particle. These inferences form the basis for simulating the damage of a carbon composite struck by a high-speed particle.

\section{B. Simulating the damage of TPS materials using LPM}

Simulations using carbon composites as the target surface is discussed in this section. The carbon composite of interest in this study is a PICA-like composite comprising of FiberForm (carbon fibers) and phenolic resin, which is highly porous ( $80 \%$ void fraction). The mechanical properties of the fiber and resin is shown in Table 1 A borosilicate (Pyrex) particle is used as the projectile with mechanical properties listed in Table 1. For the initial set of simulations discussed in this section, the particle fracture strength is set to a high value, and therefore, it is not allowed to fracture. A total of four simulations are performed with two different particle diameters and incoming velocities. The simulation parameters are shown in Table2 The simulations are performed in a manner similar to the results discussed in Sec. III.A The particle is initialized at a distance close to the surface of the target. As the simulation proceeds, the impacting force of the particle stretches the voxel bonds of the target material damaging the material as it penetrates through the composite material.

Table 2 Particle diameter and velocity for the four cases examined to study the damage process on carbon composites.

\begin{tabular}{|c|c|c|}
\hline Cases & $\begin{array}{c}\text { particle } \\
\text { diameter } \\
(\mu \mathrm{m})\end{array}$ & $\begin{array}{c}\text { velocity } \\
(\mathrm{m} / \mathrm{s})\end{array}$ \\
\hline Case 1 & 10 & 1000 \\
\hline Case 2 & 10 & 4000 \\
\hline Case 3 & 5 & 1000 \\
\hline Case 4 & 5 & 4000 \\
\hline
\end{tabular}

Figure 7 indicates four snapshots of the simulation at various times demonstrating the penetration of the particle into the material. Similar to the previous section, the voxels are colored by the damage parameter, with the red color depicting voxels (fiber and resin chunks) flying away from the base composite material. The images indicate significant damage to the material as the particle penetrates through it with the damage propagating along the entire length of the 


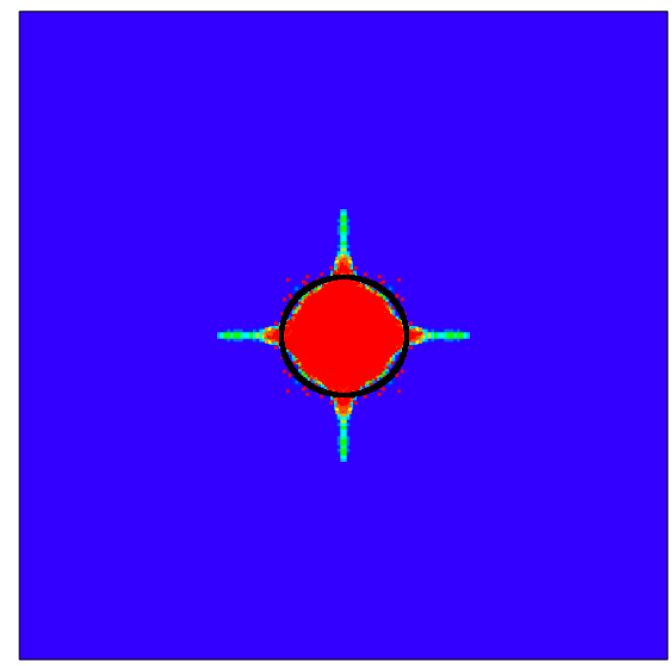

(a) Case v303,f500,nd

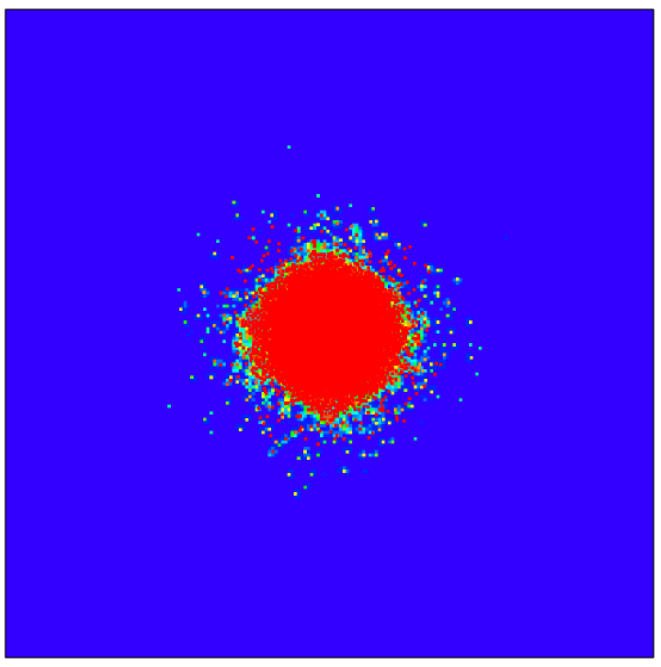

(c) Case v303,f500,d

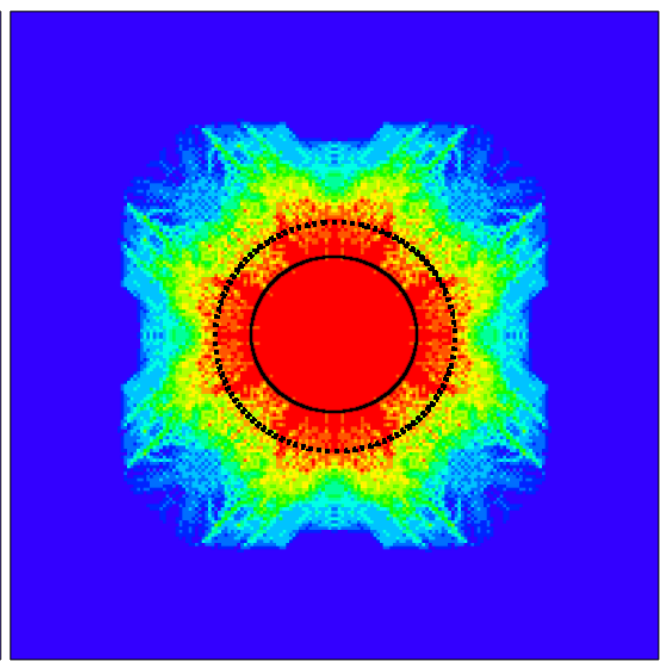

(b) Case v303,f50,nd

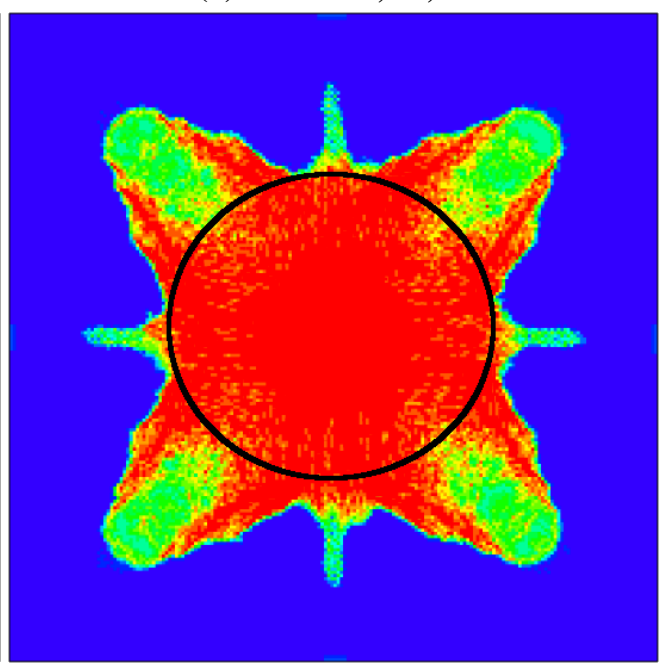

(d) Case v303,f50,d

Fig. 5 The figures show the top view of the fused silica slab after impact by the particle. Each case is obtained using different combinations of LPM model parameters discussed in Sec. II.B.1. The acronyms above are as follows: $v$ represents particle velocity, $f$ represents fracture strength of the target, $d$ implies that the particle fracture is included, nd represents that the particle fracture is not included. Therefore, the case v303,f500,nd (Fig. 5a) represents an incoming particle velocity of $303 \mathrm{~m} / \mathrm{s}$ that strikes the fused silica target with a fracture strength of $500 \mathrm{MPa}$, where the particle does not fracture upon impact, and the fused silica fractures as soon as the failure criteria is reached.

fiber in some cases. Further, chunks of voxels appear to fly away during damage that likely enter the boundary layer during the entry process. The top views of the final damaged microstructure for all four cases are shown in Fig. 8 We see a strong correlation between the impacting velocity and the damaged size. The hole created by the impact at 4 $\mathrm{km} / \mathrm{s}$ (Figs. $8 \mathrm{~b}$ and $8 \mathrm{~d}$ ) is significantly bigger than the damage caused by the particle traveling at $1 \mathrm{~km} / \mathrm{s}$ (Figs. 8a and 8c). A particle traveling with higher kinetic energy is expected to damage the target material to a greater extent. The penetration depth for each case is shown in Fig. 9, where the velocity of the particle is plotted as a function of the 




(a) Case v303,f500,nd

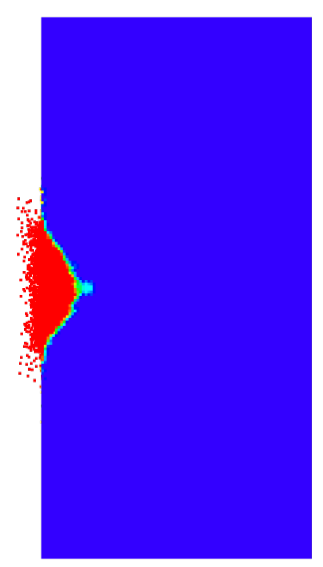

(c) Case v303,f500,d

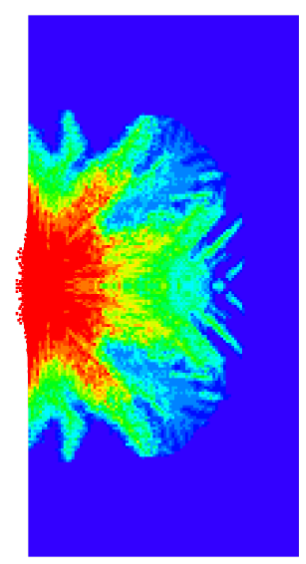

(b) Case v303,f50,nd

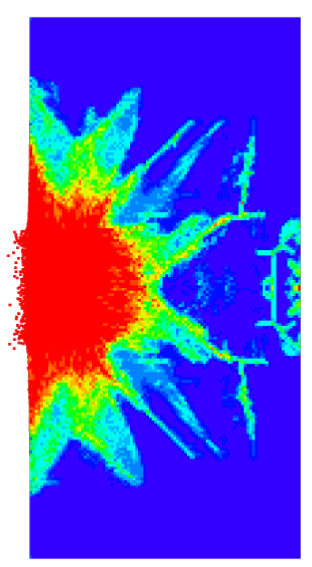

(d) Case v303,f50,d

Fig. 6 Cross-section view for the cases shown in Fig. 5 for fused silica.

penetration depth into the material. The step function profiles seen in Fig. 9 is a consequence of the porosity of the composite material. As the particle strikes a fiber or resin, it instantaneously slows down. However, once the particle overcomes the initial damage, it moves in free space until it encounters the next fiber or resin phase, instantaneously slowing down further before continuing to penetrate through the material. The particle penetrates completely through the microstructure for all cases except case $4\left(\mathrm{~d}_{p}=5 \mu \mathrm{m}, \mathrm{v}=1 \mathrm{~km} / \mathrm{s}\right)$. For this case, the particle penetrates roughly 100 $\mu m(0.1 \mathrm{~mm}$ ) into the material (Fig. 9c). We note here that the simulation with a particle diameter of $5 \mu \mathrm{m}$ and $4 \mathrm{~km} / \mathrm{s}$ did not complete because of time constraints on the compute cluster. However, based on the velocity of the particle at a depth of $150 \mu \mathrm{m}$ (Fig. 9d), it would completely penetrate through the microstructure. The length of the microstructure chosen for this study is $0.2 \mathrm{~mm}(200 \mu \mathrm{m})$, which is clearly not sufficient to bring particles striking at $4 \mathrm{~km} / \mathrm{s}$ or with a particle diameter of $10 \mu \mathrm{m}$ to a complete stop when the particle is not allowed to fracture. This length was chosen based on a previous study that analyzed the REV required to compute material permeability [34]. Since our objective in this 
article is to build a fundamental understanding on how the damage is initiated, and the influence of a damaged region on material permeability, this REV is deemed sufficient for this study. Larger size REVs are required to quantify the penetration depth if the particle is not allowed to fracture. However, as discussed below, allowing the particle to fracture results in penetration depths that are at least an order of magnitude smaller.
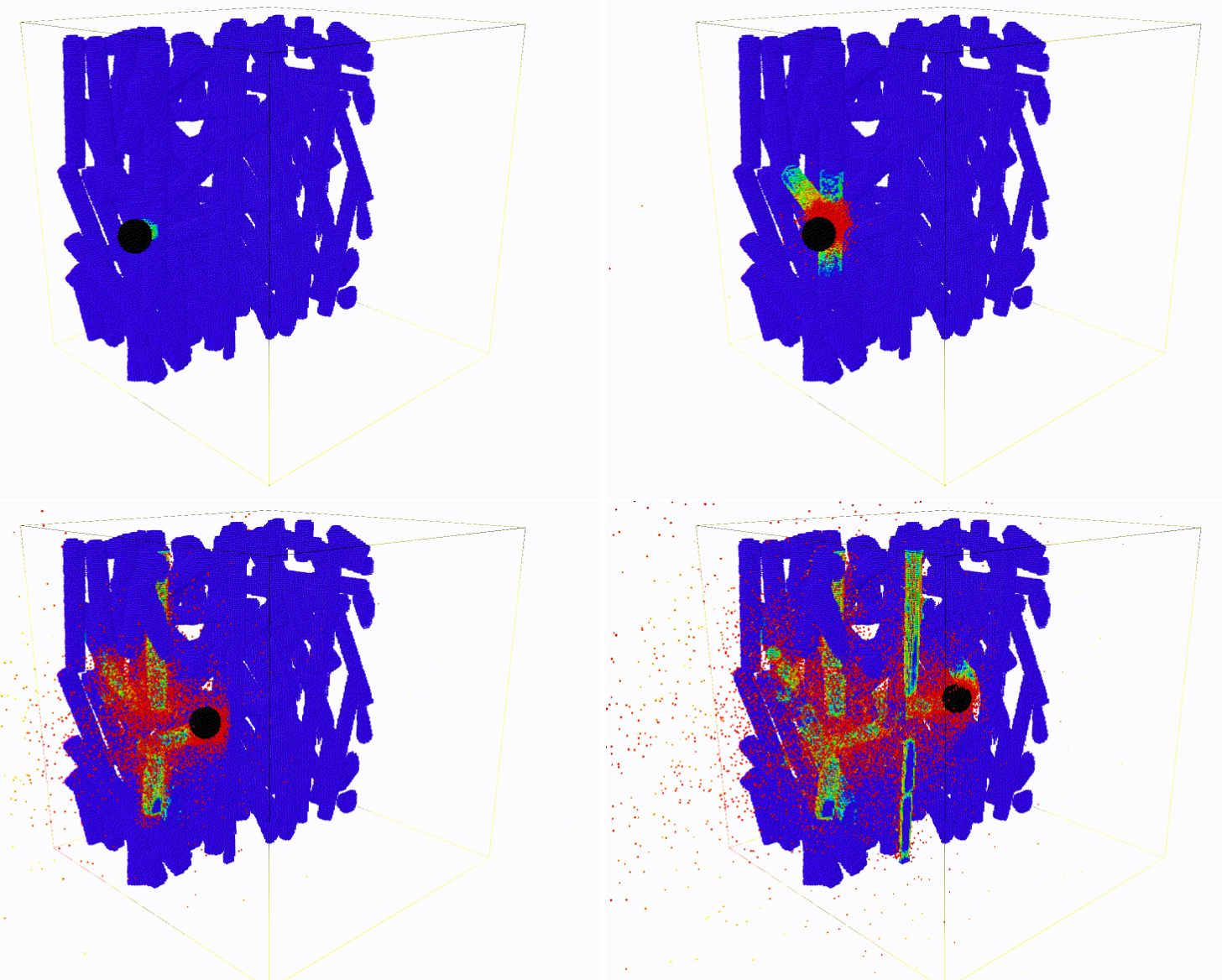

Fig. 7 Snapshots of particle penetrating through composite microstructure. The solid black voxels represent the particle. The voxels are colored based on the damage parameter (Eqn. 12), where red voxels have broken away from the composite.

In the simulations described above, the particle was not allowed to fracture upon impact with the microstructure. This assumption was deemed reasonable based on the comparisons of simulations and experiments of fused silica targets. Reasonable agreement with experimental data was found with a fracture strength of the target at $50 \mathrm{MPa}$ irrespective of the assumption used in modeling the fracture of the particle. A fracture strength of $50 \mathrm{MPa}$ for fused silica is $\sim 5$ times lower than the fracture strength of the particle (borosilicate; $280 \mathrm{MPa}$ ). However, the composite contains two phases: fibers and resin. The resin has a much lower fracture strength of $40 \mathrm{MPa}$ but the carbon fiber has a fracture strength that varies between 300 to $1000 \mathrm{MPa}$ (see Table 1). Therefore, it is likely that the inclusion of the particle fracture would influence the final damaged microstructure for carbon composites. This, in turn, will affect the overall penetration 


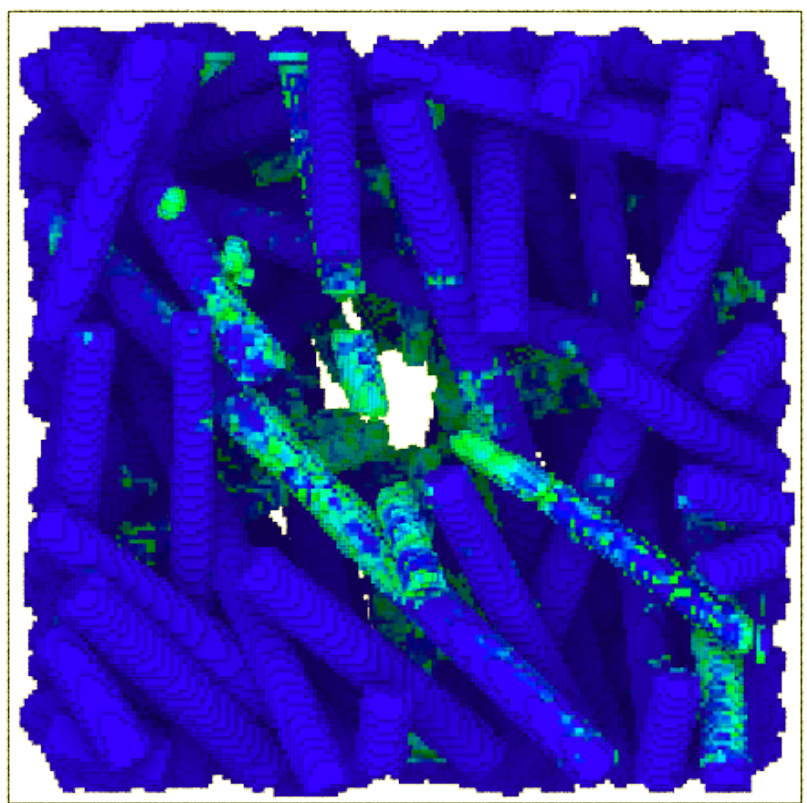

(a) Case 1: $d_{p}=10 \mu m, v=1 \mathrm{~km} / \mathrm{s}$.

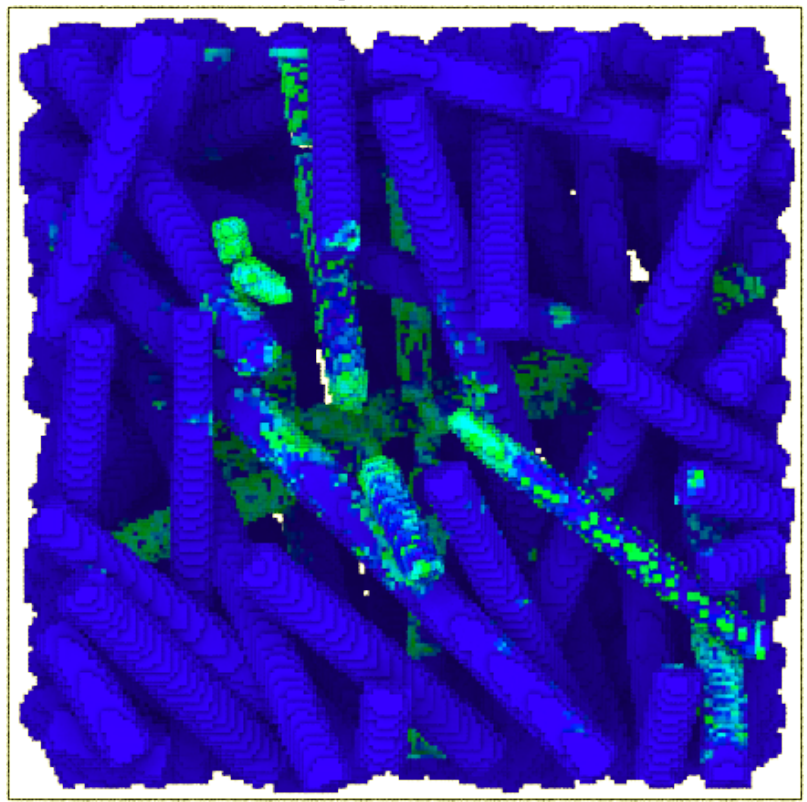

(c) Case 3: $d_{p}=5 \mu m, v=1 \mathrm{~km} / \mathrm{s}$.

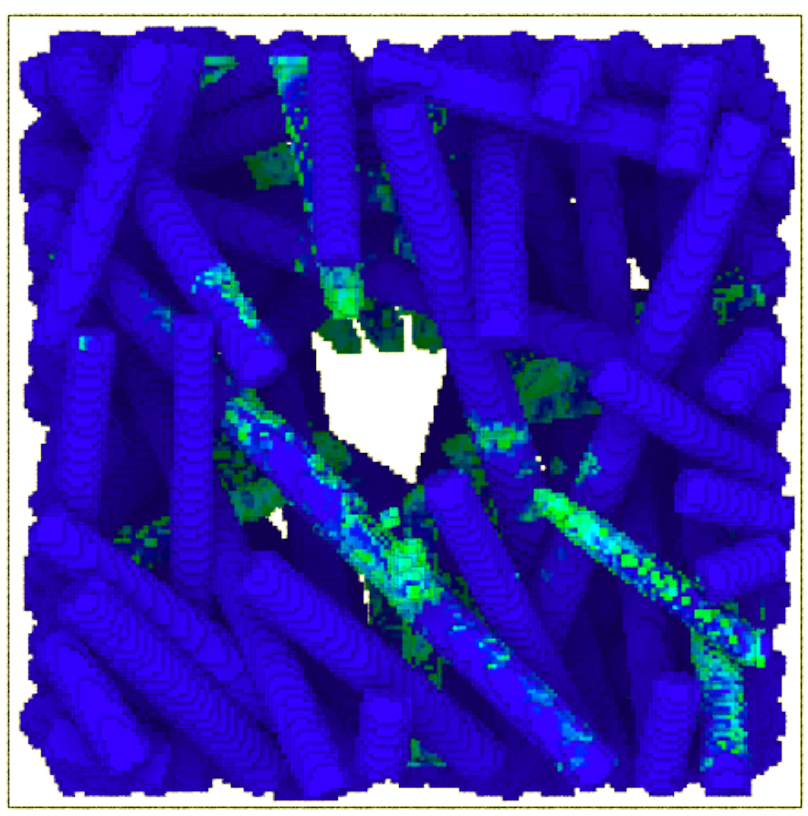

(b) Case 2: $d_{p}=10 \mu \mathrm{m}, v=4 \mathrm{~km} / \mathrm{s}$.

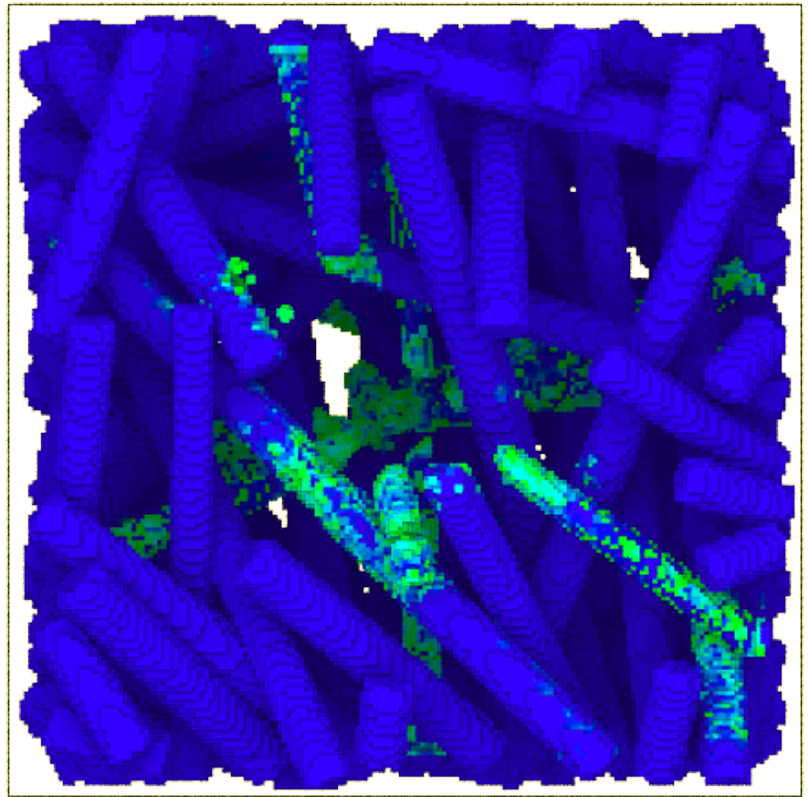

(d) Case 4: $d_{p}=5 \mu \mathrm{m}, v=4 \mathrm{~km} / \mathrm{s}$.

Fig. 8 Top view of the damaged microstructure for the four cases shown in Table 2. The simulation shown in Fig. 8d did not complete because of time constraints on the compute cluster but it is still presented because of useful insights available for comparison.

depth of particles into the microstructures of carbon composites. To understand the influence of particle fracture on penetration depth in composite materials, the impact of a borosilicate particle at $1 \mathrm{~km} / \mathrm{s}$ with a diameter of $10 \mu m$ and a fracture strength of $280 \mathrm{MPa}$ is simulated, with the fracture strength for the fibers in the carbon composite set to 330 $\mathrm{MPa}$ and $1000 \mathrm{MPa}$. The top view of the damaged microstructure for the two cases is shown in Fig. 10 . It is observed 




(a) Case 1: $d_{p}=10 \mu \mathrm{m}, v=1 \mathrm{~km} / \mathrm{s}$.

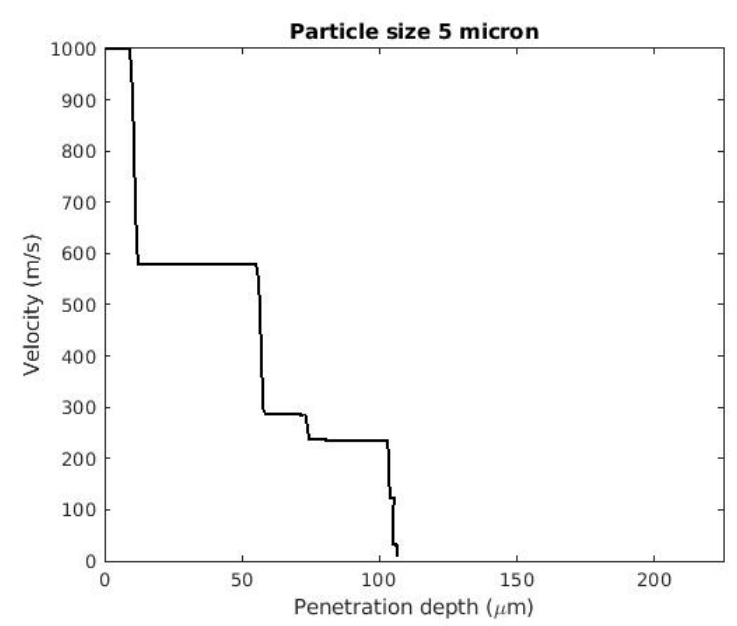

(c) Case 3: $d_{p}=5 \mu \mathrm{m}, v=1 \mathrm{~km} / \mathrm{s}$.

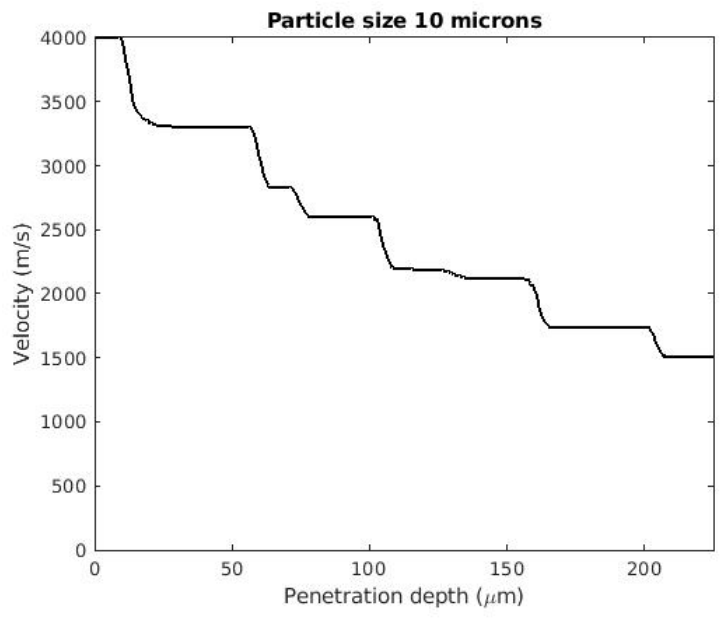

(b) Case 2: $d_{p}=10 \mu \mathrm{m}, v=4 \mathrm{~km} / \mathrm{s}$.

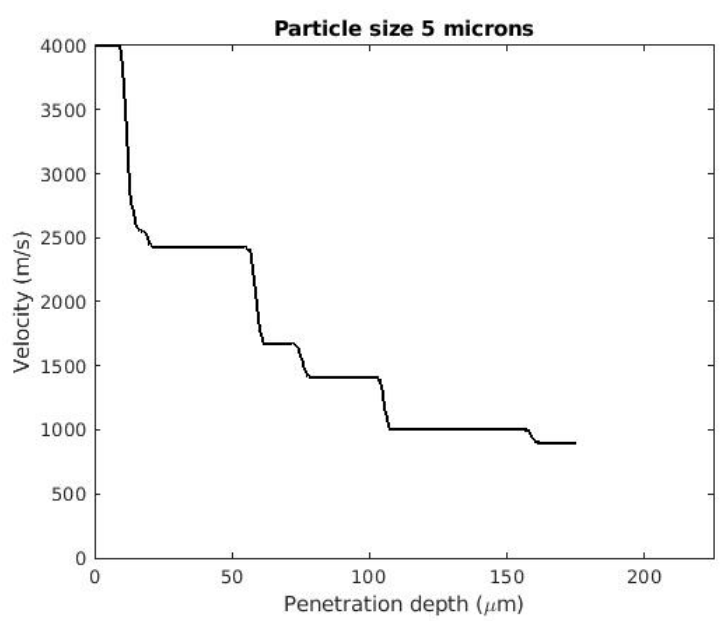

(d) Case 4: $d_{p}=5 \mu \mathrm{m}, v=4 \mathrm{~km} / \mathrm{s}$.

Fig. 9 Velocity of the particle as a function of penetration depth inside the microstructure for the four cases shown in Table 2 .

that the damage to the carbon composite is now significantly lower. With a fracture strength of $1000 \mathrm{MPa}$, only one fiber is broken (Fig. 10a). When the fracture strength is reduced to $330 \mathrm{MPa}$, roughly two fibers are broken (Fig. 10b). Based on this observation, we estimate that the damage is approximately $10-20 \mu m$ deep, which is approximately an order of magnitude lower compared to the penetration depth obtained when the particle is not allowed to fracture. This observation is in contrast to the results obtained for the solid fused silica target case (Sec. III.A), where allowing the particle to fracture did not significantly influence the crater depth. The comparison of damaged regions formed on the silica surface and carbon composite indicates that the physical processes resulting in the damage on a solid target is significantly different than the damage caused on porous composite materials. More importantly, the assumptions used for modeling the damage on flat surfaces may not be valid for modeling the damage of porous composite materials. The underlying structural processes resulting in these differences will be a subject of future study. 


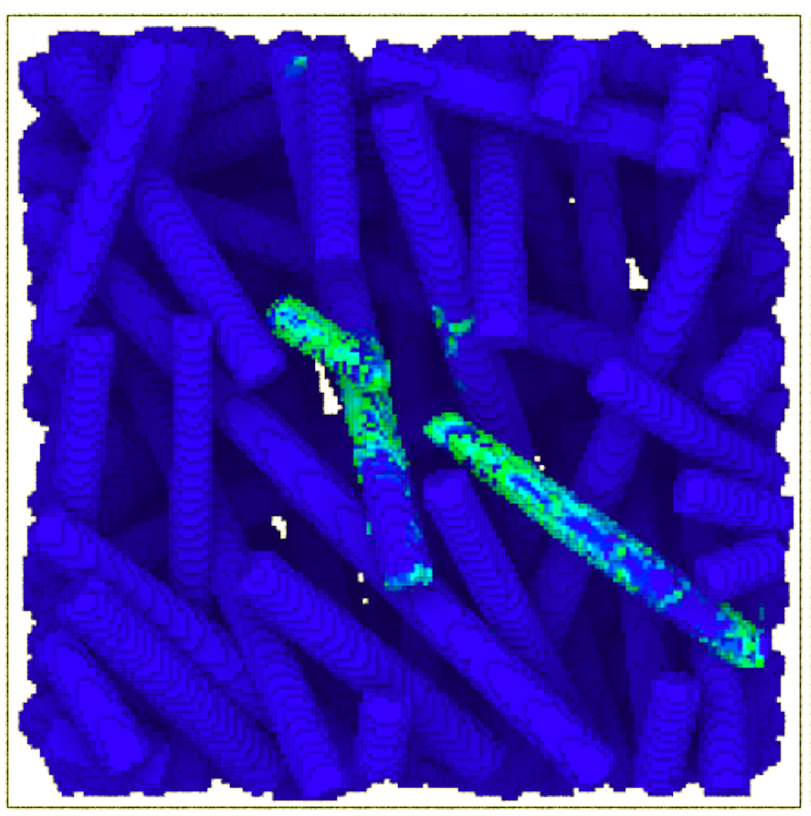

(a) Fracture strength of carbon fiber $=1000 \mathrm{MPa}$.

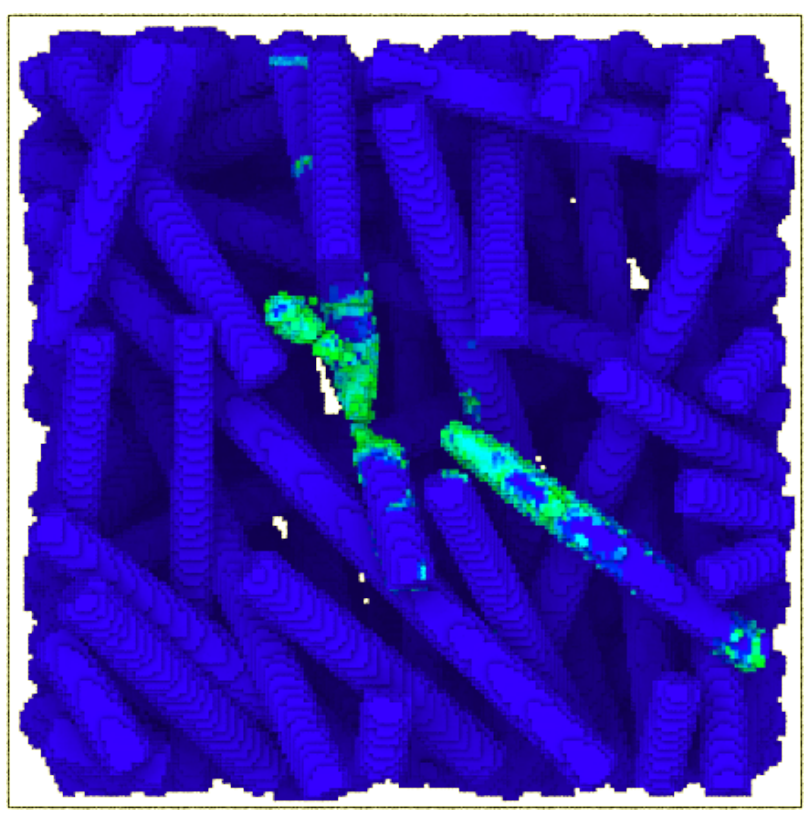

(b) Fracture strength of carbon fiber = $330 \mathrm{MPa}$.

Fig. 10 Top view of the damaged microstructure allowing the particle to fracture. Color indicates damage and fully damaged voxels are removed. A particle with a diameter of $10 \mu \mathrm{m}$ and $1 \mathrm{~km} / \mathrm{s}$ is used in both cases.

Although a quantitative model to capture the penetration depth and crater diameter as a function of particle diameter and velocity is not available from this study, qualitative insights can still be inferred. First, we see that the crater diameter on the composite is much smaller than the crater diameter on fused silica. For a particle with a diameter of $10 \mu m$ and 1 $\mathrm{km} / \mathrm{s}$, the model from Papadopoulos et al. (Eqns. 1 and 2) built using fused silica data would predict a crater diameter of $113 \mu \mathrm{m}$, while the diameter of the crater on a carbon composite predicted from our simulations is $\sim 10 \mu \mathrm{m}$. We do note here that the damaged diameter could be larger if the affected surrounding regions are taken into account. The biggest variation is seen in the penetration depth, where the Papadopoulos model provides a depth of only $3 \mu \mathrm{m}$, while the simulations predict a penetration depth of 10-20 $\mu \mathrm{m}$ when the particle is allowed to fracture, and $100 \mu \mathrm{m}$ when the particle is not allowed to fracture. For the case with a particle diameter of $10 \mu \mathrm{m}$ and $4 \mathrm{~km} / \mathrm{s}$, the penetration depth from the model of Papadopoulos is $7.6 \mu \mathrm{m}$, while the penetration depth predicted from our study when the particle is not allowed to fracture is greater than $200 \mu \mathrm{m}$ (particle completely penetrates through the material for this case). This observation is not surprising because the Flaherty experiments that were used by Papadopoulos et al. were performed with particle diameters of $O(1 \mathrm{~mm})$. Further, the model is built using experiments performed on a flat fused silica surface and not a carbon composite material. We perform this comparison because the Papadopoulos model has been historically used for carbon composites, even if it may not be valid.

The results are also compared with the empirical relation developed for Norcoat Liége [6], which is a carbon composite made of cork and phenolic resin. The crater diameter and depth obtained for a particle diameter of $10 \mu \mathrm{m}$ 
and $1 \mathrm{~km} / \mathrm{s}$ using the empirical relation for Norcoat Liége are $38.17 \mu \mathrm{m}$ and $19.08 \mu \mathrm{m}$, respectively. The damaged depth predicted by the LPM simulations when the particle is allowed to fracture is close to the experimental values of Norcoat Liége. However, we note here that only a qualitative comparison is valid here. A quantitative comparison of correlations developed from Norcoat Liége with LPM simulations discussed in this article is not apt for the following three reasons. First, the microstructure of Norcoat Liége is fundamentally different than the simulated composite. Norcoat Liége is made of cork infused with phenolic resin with a density of $470 \mathrm{~kg} / \mathrm{m}^{3}$, while the density of a PICA-like composite simulated in this article is $210 \mathrm{~kg} / \mathrm{m}^{3}$ resulting in much higher porosity for the simulated composite. Further, the fibers in the PICA-like composite have a preferential orientation in the through-the-thickness direction [33] causing the particles to strike the fiber along the radial direction as opposed to the axial direction making it easier to damage a fiber by striking it along the radial vector. The orientation of fibers in Norcoat Liége is unknown. Second, the experiments on Norcoat Liége used boron nitride and alumina as the projectiles [55] and not borosilicate. Finally, Norcoat Liége was undergoing thermochemical ablation during the experiments [55] and the expulsion of pyrolysis gases could have further reduced the velocity of impact, as well as added additional drag on the particle as it penetrated through the material. These effects are not modeled in the current study.

\section{Influence of damage on effective material permeability}

Material permeability is an important property of porous heat shields because it dictates the transport of pyrolysis gases, and the diffusion of boundary layer gases into the char layer. For entry conditions at high altitudes and typical pore sizes of the heat shield material $(40-50 \mu \mathrm{m})$, the flow inside the material could be in the non-continuum regime [56]. Therefore, a Klinkenberg formulation shown in Eqn. 14 is used for material permeability.

$$
K=K_{o}\left(1+\frac{b}{P}\right)=F
$$

In Eqn. 14, $\mathrm{K}$ is the effective material permeability, $\mathrm{K}_{o}$ is the intrinsic permeability, $\mathrm{P}$ is the pressure, and $\mathrm{b}$ is a parameter that depends on temperature and the gaseous species percolating through the material. Marschall and Milos demonstrated that K equals F (see Eqn. 13 for definition of F) [57], and the derivation can be found in Refs. [7, 57, 58]. F is referred to as the permeability force since it has units of Newton, and we use the same terminology here. The setup for the permeability simulations has been discussed in Sec. II.C. Simulations are performed at three temperatures of 300,600 , and $1000 \mathrm{~K}$. For each temperature, the specified inflow and outflow pressure at the boundary is varied from 250 to 3000 Pa with a nominal pressure difference of $\sim 100 \mathrm{~Pa}$. Simulations are run until steady state using air as

the test gas. The pressure across the sample $\left(P_{1}\right.$ and $\left.P_{2}\right)$ is extracted to compute $\Delta P=P_{2}-P_{1}, P_{\text {avg }}=\frac{1}{2}\left(P_{1}+P_{2}\right)$, and the mass flow rate $\dot{m}$ is also extracted from the simulations. The force is computed by substituting $\Delta P$ and $\dot{m}$ into Eqn. 13 and plotted against $P_{\text {avg }}$. Simulation parameters such as cell divisions, timestep, and the number to samples 
collected for averages are set based on the conclusions from the previous study on permeability computations [34]. Permeability computations are performed for the virgin microstructure and the four damaged microstructures discussed in Sec. III.B where the particle is not allowed to fracture.

The results for the permeability computations along with the change in $\mathrm{F}$ is shown in Fig. 11. We begin with our focus on the permeability force for the virgin microstructure. It has been shown previously that the difference in the simulated permeability and experimental measurements is less than 5\% [34] for the virgin microstructure. The permeability force increases with temperature because a higher value of $\mathrm{F}$ implies that it is easier for gases to percolate through the sample. F increases non-linearly with temperature (Fig. 11a because of two complementary effects. First, F is directly proportional to temperature. As temperature increases, the mean thermal speed and inter-molecular collision distances for the gas molecules increase. Consequently, it is easier for gas molecules to traverse the complex path through the porous material which reduces the pressure gradient. Second, F is inversely proportional to the pressure gradient, thereby further increasing F. However, the increase in $\mathrm{F}$ is not quadratic because the mass flow rate decreases with temperature.

Since an increase in F corresponds to an easier path for the gas molecules to traverse the microstructure, the permeability force for all damaged microstructures should be higher than the virgin microstructure because the damaged microstructure would be more porous. The higher permeability force for all damaged microstructures can be seen from Figs. 11a 11c and 11e The increase in $\mathrm{F}$ for all the damaged microstructures compared to the virgin microstructure as a percentage is shown in Figs. 11b, 11d, and 11f As expected, for a particle with a diameter of $10 \mu m$, the percentage increases with a higher velocity impact because more fibers are damaged by the impact. For an incoming particle velocity of $4 \mathrm{~km} / \mathrm{s}$, a $16 \%$ and $20 \%$ increase in $\mathrm{F}$ is obtained for a temperature of $1000 \mathrm{~K}$ and average pressures of 250 and $3000 \mathrm{~Pa}$, respectively. The percentage increase in $\mathrm{F}$ also corresponds to an increase in flow temperature of the test gas for this case (Fig. 11b). However, a similar trend is not observed for the microstructure damaged by the $1 \mathrm{~km} / \mathrm{s}$ impact (dashed lines in Fig. 11b). At $300 \mathrm{~K}$, the increase in $\mathrm{F}$ as a percentage is higher at $2000 \mathrm{~Pa}$ compared to 3000 Pa. Additionally, this increase at $300 \mathrm{~K}$ is higher than the increase at both 600 and $1000 \mathrm{~K}$. Similarly, a dip in the increase is seen for $600 \mathrm{~K}$ at $500 \mathrm{~Pa}$. No discernable trends are obtained from the percentage increase in $\mathrm{F}$ as a function of temperature or pressure for damaged microstructures that were struck by particles with a diameter of $10 \mu m$ and velocities of 1 and $4 \mathrm{~km} / \mathrm{s}$. On average, the increase in $\mathrm{F}$ as a percentage is approximately 0.4 times lower for case 1 compared to case 2 (see Table 2 for conditions). When the particle diameter is halved (10 $\mu \mathrm{m}$ to $5 \mu \mathrm{m}$ ), the increase in $\mathrm{F}$ as a percentage is also roughly reduced by a factor of 2 (see Figs.11b and 11d for comparison). Further, as the incoming velocity for particles with a diameter of $5 \mu \mathrm{m}$ is reduced from $4 \mathrm{~km} / \mathrm{s}$ to $1 \mathrm{~km} / \mathrm{s}$, the change in $\mathrm{F}$ is relatively small (see Figs. 11d and 11f for comparison). Based on the top view of the damaged microstructures (Figs. $8 \mathrm{a}$ and $8 \mathrm{c}$ ) and the bigger hole that is generated on the microstructure damaged by a particle of $10 \mu m$, one would expect a higher difference in permeability between these cases because of the new porosity of the material. However, porosity is not connected to 
the material permeability and two materials with the same porosity can have markedly different permeability values [7].

The computations of effective material permeability of four damaged microstructures demonstrates that the maximum increase in $\mathrm{F}$ is within $22 \%$. This level of change is within the variability observed for these porous composites. The permeability of the virgin material can differ by as much as $25 \%$ with minor variations in the mean fiber diameter, orientation angles, and even porosity [7, 34]. For example, a change in porosity from 0.902 to 0.887 resulted in a $19 \%$ variation in effective material permeability [34]. This initial comparison indicates that effective material permeability is primarily dictated by the fiber radii and orientations within the microstructure, and increases in effective permeability through damage is within the manufacturing variability. However, it should be noted that this increase from damage is an additive factor to the underlying bounds to the permeability values resulting from the manufacturing variability.

\section{Conclusions}

Simulations of particles striking a material at high speeds were performed using a novel computational technique called the lattice particle method (LPM) to understand the initiation and growth of craters formed upon impact. The lattice particle method was initially used to study the impact of a borosilicate particle striking a solid fused silica target and the results were compared with experiments. A parametric study on the effect of the material properties on the damage was performed as part of the comparison. It was found that the fracture strength of the silica surface primarily dictated the damage patterns found on the target. When the fracture strength of the target was $500 \mathrm{MPa}$, small diamond-like damage patterns were obtained on the surface while a fracture strength of $50 \mathrm{MPa}$ resulted in petaloid structures. The crater diameter and depth were also quantified, and comparison with experiments indicated good qualitative agreement when the fracture strength of the fused silica surface was set to $50 \mathrm{MPa}$. Investigation on allowing the particle to fracture during the damage process indicated that it influenced the damage patterns but the damage diameter and depth were comparable to the case where the particle was not allowed to fracture.

The LPM technique was then used to simulate the damage process on microstructures of porous carbon composites. The microstructures were digitally generated using an in-house code that has been shown to reproduce features of the real material in past studies. Simulations indicated that the particle penetrates over $200 \mu \mathrm{m}$ into the microstructure when the particle diameter is $10 \mu \mathrm{m}$ with velocities of $1 \mathrm{~km} / \mathrm{s}$ and $4 \mathrm{~km} / \mathrm{s}$, as well as $5 \mu m$ with a velocity of $4 \mathrm{~km} / \mathrm{s}$ when the particle was not allowed to fracture. However, in contrast to simulations of fused silica cases, the penetration depth decreased by at least an order of magnitude to $20 \mu \mathrm{m}$ when the particle was allowed to fracture. The damaged microstructures were imported into a direct simulation Monte Carlo flow solver and effective permeability of the virgin and damaged microstructures were compared. It was found that the maximum increase in permeability force was only $20 \%$ indicating that the hole created in the microstructure by particle impact did not significantly influence the path traversed by gases percolating through the material. Future work will involve detailed comparisons with experiments of Norcoat Liége by including the expulsion of pyrolysis gases through the microstructure while the damage is occurring, 
and constructing new correlations for a PICA-like composite.

\section{Acknowledgements}

The authors would like to acknowledge the financial support through NASA Kentucky EPSCoR award Grant Number 80NSSC19M0052. Poovathingal was also supported in part by NASA award Grant number 80NSSC20K1072. The authors also thank the University of Kentucky Center for Computational Sciences and Information Technology Services Research Computing for their support and use of the Lipscomb Compute Cluster and associated research computing resources.

\section{References}

[1] Pollack, J. B., Colburn, D. S., Flasar, F. M., Kahn, R., Carlston, C., and Pidek, D., "Properties and effects of dust particles suspended in the Martian atmosphere," Journal of Geophysical Research: Solid Earth, Vol. 84, No. B6, 1979, pp. $2929-2945$.

[2] Toon, O. B., Pollack, J. B., and Sagan, C., "Physical properties of the particles composing the Martian dust storm of 1971-1972," Icarus, Vol. 30, No. 4, 1977, pp. 663-696.

[3] Anderson, E., and Leovy, C., "Mariner 9 television limb observations of dust and ice hazes on Mars," Journal of the Atmospheric Sciences, Vol. 35, No. 4, 1978, pp. 723-734.

[4] Chu, D. A., Kaufman, Y., Zibordi, G., Chern, J., Mao, J., Li, C., and Holben, B., "Global monitoring of air pollution over land from the Earth Observing System-Terra Moderate Resolution Imaging Spectroradiometer (MODIS)," Journal of Geophysical Research: Atmospheres, Vol. 108, No. D21, 2003.

[5] Carroll, M., “The Gas and Ice Giants,” Living Among Giants, Springer, 2015, pp. 46-67.

[6] Palmer, G., Ching, E., Ihme, M., Allofs, D., and Gülhan, A., "Modeling Heat-Shield Erosion due to Dust Particle Impacts for Martian Entries," Journal of Spacecraft and Rockets, Vol. 57, No. 5, 2020, pp. 857-875.

[7] Poovathingal, S., Stern, E. C., Nompelis, I., Schwartzentruber, T. E., and Candler, G. V., "Nonequilibrium flow through porous thermal protection materials, Part II: Oxidation and pyrolysis,” Journal of Computational Physics, Vol. 380, 2019 , pp. 427-441.

[8] Bowman, W. H., and Lawrence, R. M., "Space Resource. Ablative materials for high-temperature thermal protection of space vehicles," Journal of Chemical Education, Vol. 48, No. 10, 1971, p. 690.

[9] Papadopoulos, P., Tauber, M. E., and Chang, I.-D., "Heatshield erosion in a dusty Martian atmosphere," Journal of Spacecraft and Rockets, Vol. 30, No. 2, 1993, pp. 140-151.

[10] Palmer, G., Chen, Y.-K., Papadopoulos, P., and Tauber, M., "Reassessment of effect of dust erosion on heatshield of Mars entry vehicle," Journal of Spacecraft and Rockets, Vol. 37, No. 6, 2000, pp. 747-752. 
[11] Elangovan, R., and Cao, H., "Dusty supersonic viscous flow over a two-dimensional blunt body," Journal of thermophysics and heat transfer, Vol. 4, No. 4, 1990, pp. 529-533.

[12] Saito, T., Marumoto, M., and Takayama, K., "Numerical investigations of shock waves in gas-particle mixtures," Shock Waves, Vol. 13, No. 4, 2003, pp. 299-322.

[13] Ching, E. J., and Ihme, M., "Sensitivity study of high-speed dusty flows over blunt bodies simulated using a discontinuous Galerkin method," AIAA Scitech 2019 Forum, 2019, p. 0895.

[14] Ching, E. J., Brill, S. R., Barnhardt, M., and Ihme, M., "A two-way coupled Euler-Lagrange method for simulating multiphase flows with discontinuous Galerkin schemes on arbitrary curved elements," Journal of Computational Physics, Vol. 405, 2020, p. 109096.

[15] Schoner, R. J., CMA Manual, April 1970.

[16] Moyer, C. B., and Rindal, R. A., "An analysis of the coupled chemically reacting boundary layer and charring ablator. Part 2 Finite difference solution for the in-depth response of charring materials considering surface chemical and energy balances," Tech. Rep. NASA-CR-1061, Aerotherm Corp., 061968.

[17] Chen, Y. K., and Milos, F. S., "Ablation and Thermal Response Program for Spacecraft Heatshield Analysis," Journal of Spacecraft and Rockets, Vol. 36, No. 3, 1999, pp. 1-9.

[18] Chen, Y. K., and Milos, F., “Three-Dimensional Ablation and Thermal Response Simulation System,” 38th AIAA Thermophysics Conference, AIAA, Toronto, ????

[19] Chen, Y. K., Milos, F., and Tahir, G., "Validation of a Three-Dimensional Ablation and Thermal Response Simulation Code," 10th AIAA/ASME Joint Thermophysics and Heat Transfer Conference, AIAA, Chicago, 2010.

[20] Milos, F. S., and K, C. Y., “Two-Dimensional Ablation, Thermal Response, and Sizing Program for Pyrolyzing Ablators,” Journal of Spacecraft and Rockets, Vol. 46, No. 6, 2009, pp. 1089-1099.

[21] Amar, A. J., Blackwell, B. F., and Edwards, J. R., “One-Dimensional Ablation Using a Full Newton's Method and Finite Control Volume Procedure," Journal of Thermophysics and Heat Transfer, Vol. 22, No. 1, 2008, pp. 71-82.

[22] Amar, A. J., Blackwell, B. F., and Edwards, J. R., "Development and Verification of a One-Dimensional Ablation Code Including Pyrolysis Gas Flow,” Journal of Thermophysics and Heat Transfer, Vol. 23, No. 1, 2009, pp. 59-71.

[23] Amar, A. J., Oliver, B., Kirk, B., Salazar, G., and Droba, J., “Overview of the CHarring Ablator Response (CHAR) Code,” 46th AIAA Thermophysics Conference, AIAA, Washington, 2016, pp. 2016-33385.

[24] Martin, A., and Boyd, I. D., "Strongly coupled computation of material response and nonequilibrium flow for hypersonic ablation," Spacecraft and Rockets, Vol. 52, No. 1, 2015, pp. 89-104. 
[25] Martin, A., and Boyd, I. D., "Mesh tailoring for strongly coupled computation of ablative material in nonequilibrium hypersonic flow," 10th AIAA/ASME Joint Thermophysics and Heat Transfer Conference, AIAA, Chicago, 2010.

[26] Martin, A., and Boyd, I. D., "Non-Darcian Behavior of Pyrolysis Gas in a Thermal Protection System," Journal of Thermophysics and Heat Transfer, Vol. 24, No. 1, 2010, pp. 60-68.

[27] Weng, H., and Martin, A., "Multidimensional Modeling of Pyrolysis Gas Transport Inside Charring Ablative Materials," Journal of Thermophysics and Heat Transfer, Vol. 28, No. 4, 2014, pp. 583-597.

[28] Weng, H., Bailey, S. C. C., and Martin, A., "Numerical study of iso-Q sample geometric effects on charring ablative materials," International Journal of Heat and Mass Transfer, Vol. 80, 2015, pp. 570-596.

[29] Weng, H., and Martin, A., "Numerical Investigation of Thermal Response Using Orthotropic Charring Ablative Material," Journal of Thermophysics and Heat Transfer, Vol. 29, No. 3, 2015, pp. 429-438.

[30] Lachaud, J., and Mansour, N. N., "Porous-Material Analysis Toolbox Based on OpenFOAM and Applications," Journal of Thermophysics and Heat Transfer, Vol. 28, No. 2, 2014, pp. 191-202.

[31] Schulz, J. C., Stern, E., Muppidi, S., Palmer, G., Schroeder, O., and Martin, A., "Development of a three-dimensional, unstructured material response design tool," 55th AIAA Aerospace Sciences Meeting, 2017, p. 0667.

[32] Flaherty, R. E., "Impact characteristics in fused silica for various projectile velocities," Journal of Spacecraft and Rockets, Vol. 7, No. 3, 1970, pp. 319-324.

[33] Stern, E. C., Poovathingal, S., Nompelis, I., Schwartzentruber, T. E., and Candler, G. V., "Nonequilibrium flow through porous thermal protection materials, Part I: Numerical methods," Journal of Computational Physics, Vol. 380, 2019 , pp. $408-426$.

[34] Poovathingal, S. J., Soto, B. M., and Brewer, C., “Computation of Effective Permeability for Porous Carbon Composites,” 2021.

[35] Zhang, C., and Schwartzentruber, T. E., "Robust Cut-cell Algorithms for DSMC Implementations Employing Multi-level Cartesian Grids," Computers and Fluids, Vol. 69, 2012, pp. 122-135. doi:https://doi.org/10.1016/j.compfluid.2012.08.013.

[36] Banerjee, A., and Poovathingal, S. J., "Investigation of In-Depth penetration of Radiative Heating in Thermal Protection System (TPS)," AIAA Scitech 2021 Forum, 2021, p. 1631.

[37] Chen, H., Lin, E., Jiao, Y., and Liu, Y., “A generalized 2D non-local lattice spring model for fracture simulation,” Computational Mechanics, Vol. 54, 2014, pp. 1541-1558. doi:10.1007/s00466-014-1075-4.

[38] Chen, H., Jiao, Y., and Liu, Y., "Investigating the microstructural effect on elastic and fracture behavior of polycrystals using a nonlocal lattice particle model," Materials Science and Engineering: A, Vol. 631, 2015, pp. 173-180. doi: 10.1016/j.msea.2015.02.046.

[39] Chen, H., Meng, L., Chen, S., Jiao, Y., and Liu, Y., "Numerical investigation of microstructure effect on mechanical properties of bi-continuous and particulate reinforced composite materials," Computational Materials Science, Vol. 122, 2016, pp. $288-294$. doi:10.1016/j.commatsci.2016.05.037. 
[40] Chen, H., Xu, Y., Jiao, Y., and Liu, Y., "A novel discrete computational tool for microstructure-sensitive mechanical analysis of composite materials," Materials Science and Engineering: A, Vol. 659, 2016, pp. 234-241. doi:10.1016/j.msea.2016.02.063.

[41] Chen, H., "Constructing continuum-like measures based on a nonlocal lattice particle model: Deformation gradient, strain and stress tensors," International Journal of Solids and Structures, Vol. 169, 2019, pp. 177-186. doi:10.1016/j.ijsolstr.2019.04.014.

[42] Chen, H., and Liu, Y., "A non-local 3D lattice particle framework for elastic solids," International Journal of Solids and Structures, Vol. 81, 2016, pp. 411-420. doi:10.1016/j.ijsolstr.2015.12.026.

[43] Bacon, R., "Carbon fibers from rayon precursors," Chemistry and physics of carbon, 1973, pp. 6-7.

[44] Akato, K., "Pretreatment and pyrolysis of rayon-based precursor for carbon fibers," 2012.

[45] Krucinska, I., and Stypka, T., "Direct measurement of the axial Poisson's ratio of single carbon fibres," Composites Science and Technology, Vol. 41, No. 1, 1991, pp. 1-12.

[46] Yeh, M.-K., Tai, N.-H., and Lin, Y.-J., "Mechanical properties of phenolic-based nanocomposites reinforced by multi-walled carbon nanotubes and carbon fibers," Composites Part A: Applied Science and Manufacturing, Vol. 39, No. 4, 2008, pp. 677-684.

[47] Jin, H., Lu, W.-Y., and Nelson, K., "Mechanical Characterization of Fiber Reinforced Polymer Composites.” Tech. rep., Sandia National Lab.(SNL-CA), Livermore, CA (United States), 2014.

[48] Plimpton, S., Moore, S., Borner, A., Stagg, A., Koehler, T., Torczynski, J., and Gallis, M., "Direct simulation Monte Carlo on petaflop supercomputers and beyond," Physics of Fluids, Vol. 31, No. 8, 2019, p. 086101. doi:https://doi.org/10.1063/1.5108534.

[49] Gallis, M. A., Koehler, T., Torczynski, J. R., and Plimpton, S. J., "Direct simulation Monte Carlo investigation of the RayleighTaylor instability," Physical Review Fluids, Vol. 1, No. 4, 2016, p. 043403. doi:https://doi.org/10.1103/PhysRevFluids.1.043403.

[50] Gallis, M., Bitter, N., Koehler, T., Torczynski, J., Plimpton, S., and Papadakis, G., "Molecular-level simulations of turbulence and its decay," Physical Review Letters, Vol. 118, No. 6, 2017, p. 064501. doi:https://doi.org/10.1103/PhysRevLett.118.064501.

[51] Lorensen, W. E., and Cline, H. E., "Marching cubes: A high resolution 3D surface construction algorithm," ACM siggraph computer graphics, Vol. 21, No. 4, 1987, pp. 163-169. doi:https://doi.org/10.1145/37402.37422.

[52] Nance, R. P., Hash, D. B., and Hassan, H. A., "Role of Boundary Conditions in Monte Carlo Simulation of Microelectromechanical Systems," Journal of Thermophysics and Heat Transfer, Vol. 12, No. 3, 1998, pp. 447-449. doi:https://doi.org/10.2514/2.6358.

[53] Fang, Y., and Liou, W., "Microfluid flow computations using a parallel DSMC code," 40th AIAA Aerospace Sciences Meeting \& Exhibit, 2002, p. 1057. doi:https://doi.org/10.2514/6.2002-1057.

[54] Poovathingal, S., Schwartzentruber, T. E., Murray, V. J., and Minton, T. K., "Molecular simulation of carbon ablation using beam experiments and resolved microstructure,” AIAA journal, Vol. 54, No. 3, 2016, pp. 999-1010. 
[55] Keller, K., Lindenmaier, P., Pfeiffer, E., Esser, B., Gülhan, A., Marraffa, L., Omaly, P., and Desjean, M.-C., "Dust Particle Erosion during Mars Entry. Issue1," 40th International Conference on Environmental Systems, 2009, p. 6283.

[56] Lachaud, J., Cozmuta, I., and Mansour, N. N., "Multiscale Approach to Ablation Modeling of Phenolic Impregnated Carbon Ablators," Journal of Spacecraft and Rockets, Vol. 47, No. 6, 2010, pp. 910-921.

[57] Marschall, J., and Milos, F. S., "Gas Permeability of Rigid Fibrous Refractory Insulations,” Journal of Thermophysics and Heat Transfer, Vol. 12, No. 4, 1998, pp. 528-535.

[58] Panerai, F., White, J. D., Cochell, T. J., Schroeder, O. M., Mansour, N. N., Wright, M. J., and Martin, A., "Experimental measurements of the permeability of fibrous carbon at high-temperature," International Journal of Heat and Mass Transfer, Vol. 101, No. C, 2016, pp. 267-273.

\section{Appendix}

As mentioned previously in Sec. II.B.1, a handbook with properties for fused silica and Pyrex is not available since it varies significantly based on the manufacturer. Therefore, for both Pyrex (used as the particle) and fused silica, we rely on manufacturer data sheets to obtain representative values. The url links for the properties are given below. Properties of carbon fiber and resin are available in literature.

Fused silica

- https://www .azom.com/properties.aspx?ArticleID=1387

- https://en.wikipedia.org/wiki/Fused_quartz

- https://technicalglass.com/technical_properties/

- https://www.momentive.com/en-us/categories/quartz/mechanical-properties

- https://highlyeducatedti.com/blogs/information/thermal-shock-vs-tensile-strength Pyrex (Borosilicate glass)

- http://valleydesign.com/pyrex.htm

- http://www.mit.edu/ 6.777/matprops/pyrex.htm

- https://highlyeducatedti.com/blogs/information/thermal-shock-vs-tensile-strength 


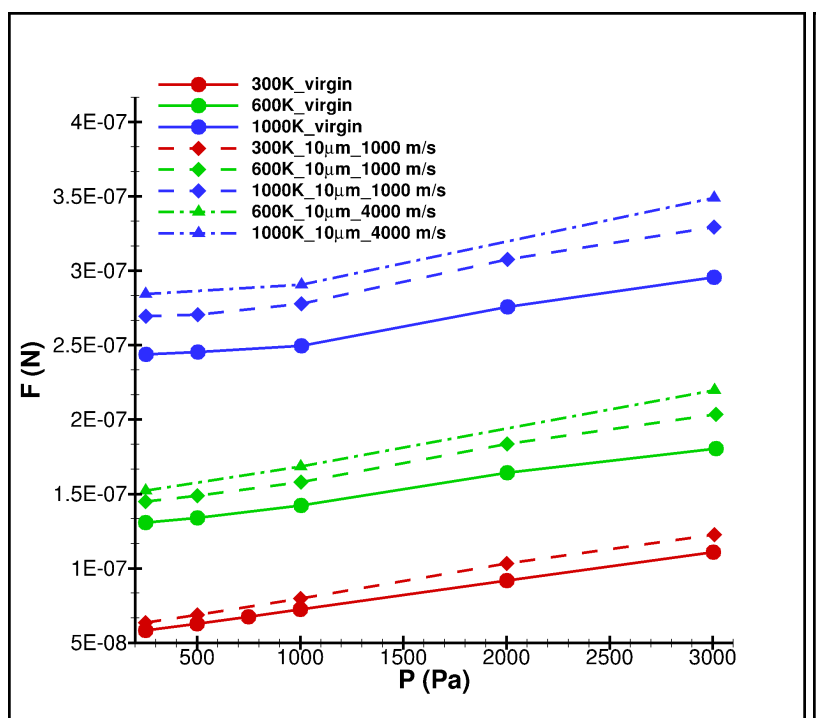

(a) Effect of changing initial velocity for $\mathbf{d}_{p}=10 \mu \mathrm{m}$.

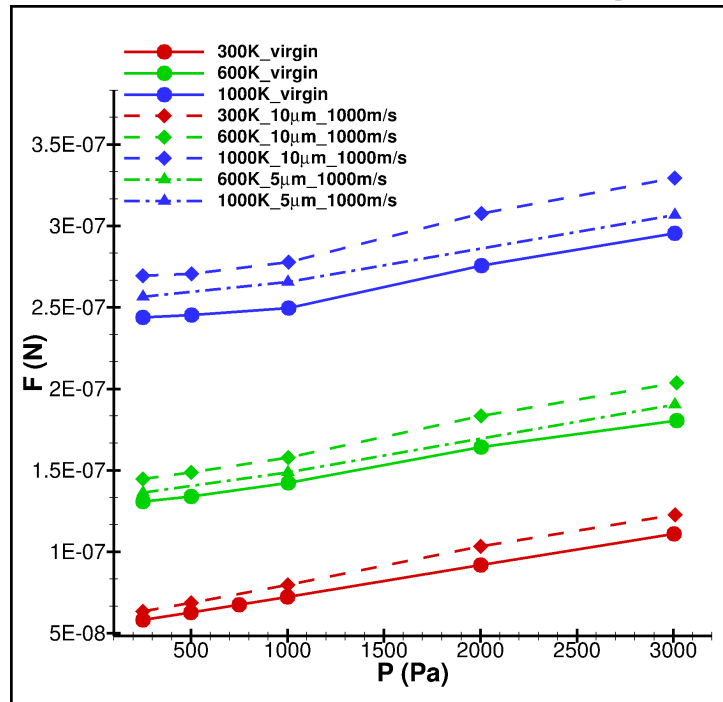

(c) Effect of changing particle size.

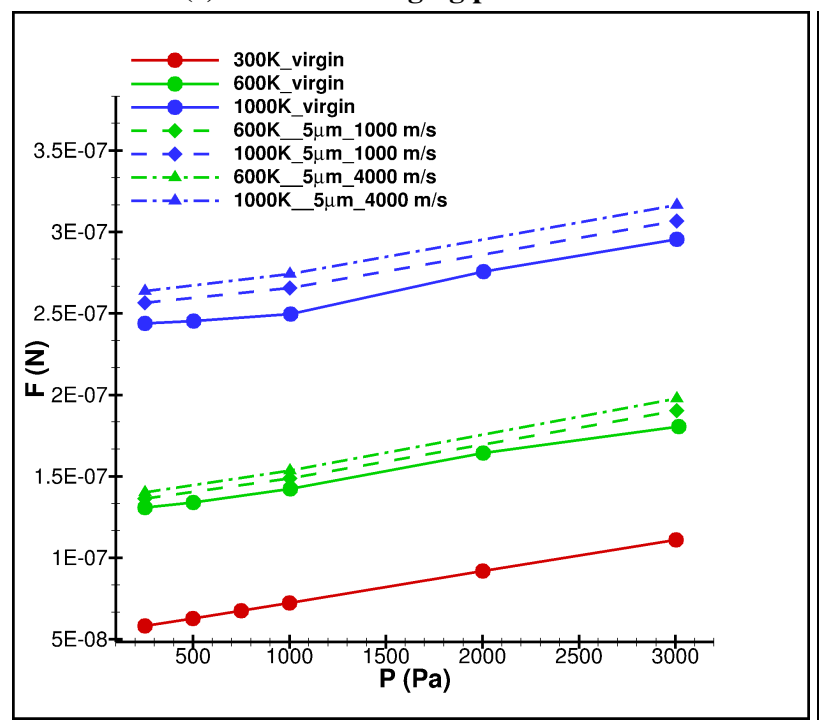

(e) Effect of changing initial velocity for $\mathbf{d}_{p}=5 \mu \mathrm{m}$.

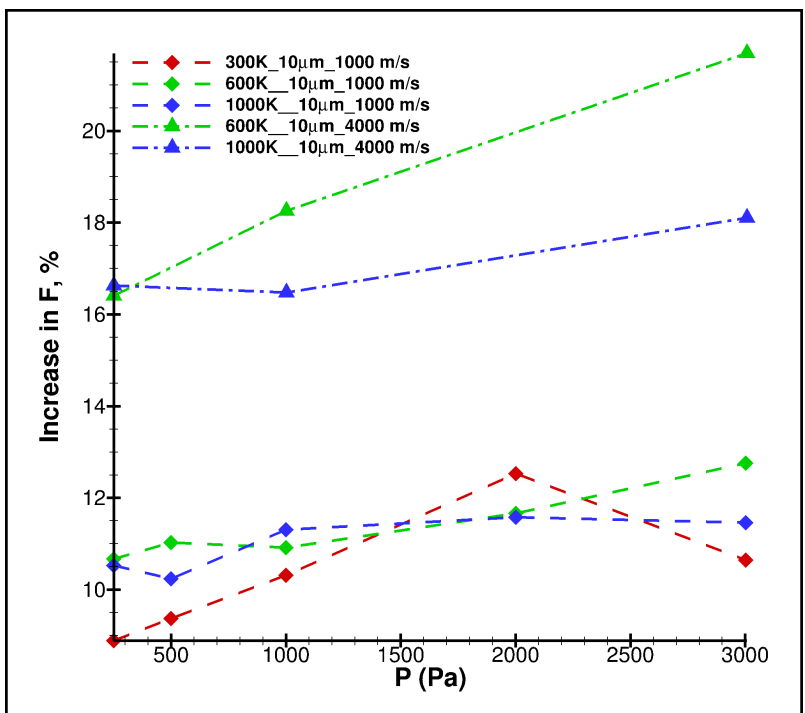

(b) Increase in F for $\mathbf{d}_{p}=10 \mu \mathrm{m}, \mathbf{v}=\mathbf{1}$ and $4 \mathrm{~km} / \mathrm{s}$.

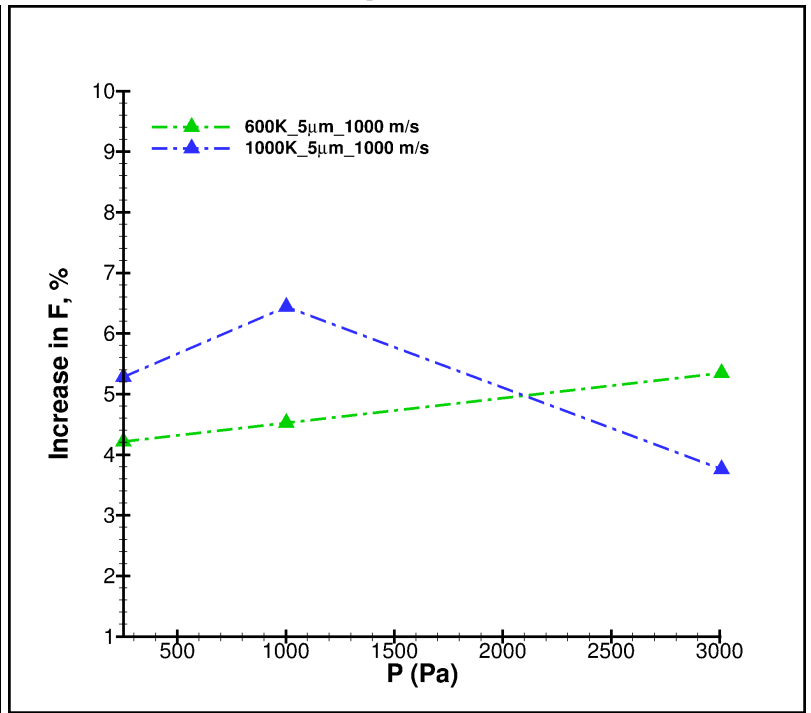

(d) Increase in $\mathbf{F}$ for $\mathbf{d}_{p}=5 \mu m, \mathbf{v}=\mathbf{1 0 0 0} \mathbf{~ m} / \mathbf{s}$.

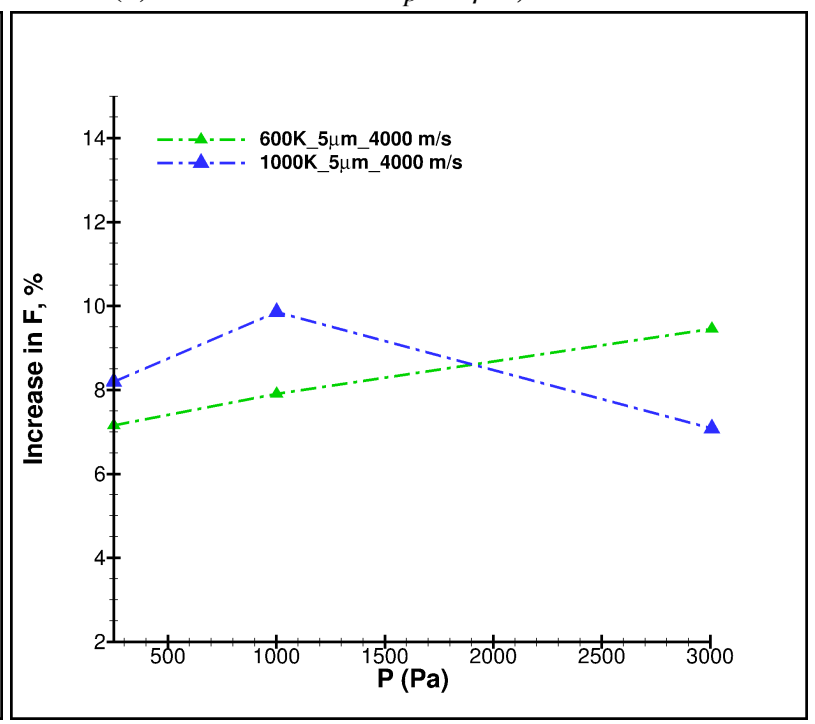

(f) Increase in $\mathbf{F}$ for $\mathbf{d}_{p}=5 \mu m, \mathbf{v}=\mathbf{4 0 0 0} \mathrm{m} / \mathrm{s}$.

Fig. 11 The figures on the left shows the permeability force $(F)$ as a function of average pressure across the sample. Figures on the right compare the change in $F$ for each case with respect to the values for the virgin microstructure. 